\title{
The Homogenized Energy Model (HEM) for Characterizing Polarization and Strains in Hysteretic Ferroelectric Materials: Implementation Algorithms and Data-Driven Parameter Estimation Techniques
}

\author{
Zhengzheng $\mathrm{Hu}$ and Ralph C. Smith \\ Department of Mathematics \\ Center for Research in Scientific Computation \\ North Carolina State University \\ Raleigh, NC 27695 \\ Jon Ernstberger \\ Department of Mathematics \\ LaGrange College \\ LaGrange, GA 30240
}

\begin{abstract}
Ferroelectric materials, such as PZT, PLZT, PMN and $\mathrm{BaTiO}_{3}$, provide unique actuator and sensor capabilities for applications including nanopositioning, high speed valves and fuel injectors, camera focusing and shutter mechanisms, ultrasonic devices for biomedical imaging and treatment, and energy harvesting devices. However, to achieve the full potential of the materials, it is necessary to develop and employ models that quantify the creep, rate-dependent hysteresis, and constitutive nonlinearities that are intrinsic to the materials due to their domain structure. The success of models requires that they be highly efficient to implement since real-time applications can require $\mathrm{kHz}$ to $\mathrm{MHz}$ rates. The calibration of models for specific materials, devices, and applications, requires efficient and robust parameter estimation algorithms. Finally, control designs can be facilitated by models that admit efficient and robust approximate inversion. The homogenized energy model (HEM) is a multiscale, micromechanical framework that quantifies a range of hysteretic phenomena intrinsic to ferroelectric, ferromagnetic and ferroelastic materials. In this paper, we present highly efficient implementation and parameter estimation algorithms for the ferroelectric model. This includes techniques to construct analytic Jacobians and data-driven algorithms to determine initial parameter estimates to facilitate subsequent optimization. The efficiency of these algorithms facilitates material and device characterization and provides the basis for constructing efficient and robust inverse algorithms for model-based control design. The model implementation, calibration, and validation are illustrated using rate-dependent PZT data and single crystal $\mathrm{BaTiO}_{3}$ data.
\end{abstract}




\section{Nomenclature}

\begin{tabular}{|c|c|}
\hline$d$ & Piezoelectric constant $(\mathrm{m} / \mathrm{V}=\mathrm{C} / \mathrm{N})$ \\
\hline$h$ & Piezoelectric constant $(\mathrm{V} / \mathrm{m}=\mathrm{N} / \mathrm{C})$ \\
\hline$E$ & Electric field $(\mathrm{V} / \mathrm{m})$ \\
\hline$g$ & Gibbs energy for dipoles (CV) \\
\hline$G_{\alpha}$ & Gibbs energy density of $\alpha$-variant $\left(\mathrm{CV} / \mathrm{m}^{3}\right)$ \\
\hline$G_{\alpha}^{e}$ & Electric Gibbs energy density of $\alpha$-variant $\left(\mathrm{CV} / \mathrm{m}^{3}\right)$ \\
\hline$P$ & Polarization $\left(\mathrm{C} / \mathrm{m}^{2}\right)$ \\
\hline $\bar{P}$ & Polarization kernel $\left(\mathrm{C} / \mathrm{m}^{2}\right)$ \\
\hline$P^{\alpha}$ & Polarization of $\alpha$-variant $\left(\mathrm{C} / \mathrm{m}^{2}\right)$ \\
\hline$P_{R}^{\alpha}$ & Remanent polarization of $\alpha$-variant $\left(\mathrm{C} / \mathrm{m}^{2}\right)$ \\
\hline$P_{m}^{\alpha}$ & Minimum polarization of $\alpha$-variant $\left(\mathrm{C} / \mathrm{m}^{2}\right)$ \\
\hline$s_{\alpha}^{E}$ & Elastic compliance of $\alpha$-variant at constant field $\left(\mathrm{m}^{2} / \mathrm{N}\right)$ \\
\hline$T$ & Temperature $(\mathrm{K})$ \\
\hline$x_{+}, x_{-}, x_{90}$ & Fraction of positively, negatively and $90^{\circ}$ oriented dipoles (Unitless) \\
\hline$Y_{\alpha}^{P}$ & Elastic stiffness of $\alpha$-variant at constant polarization $\left(\mathrm{N} / \mathrm{m}^{2}\right)$ \\
\hline$\epsilon$ & Permittivity $(\mathrm{F} / \mathrm{m}=\mathrm{C} / \mathrm{Vm})$ \\
\hline$\varepsilon$ & Strain (Unitless) \\
\hline $\bar{\varepsilon}$ & Strain kernel \\
\hline$\varepsilon^{\alpha}$ & Strain of $\alpha$-variant \\
\hline$\varepsilon_{R}^{\alpha}$ & Remanence strain of $\alpha$-variant \\
\hline$\varepsilon_{m}^{\alpha}$ & Minimum strain of $\alpha$-variant \\
\hline$\eta^{\varepsilon}$ & Inverse susceptibility at constant strain $(\mathrm{m} / \mathrm{F}=\mathrm{Vm} / \mathrm{C})$ \\
\hline$\sigma$ & Stress $\left(\mathrm{N} / \mathrm{m}^{2}\right)$ \\
\hline$\tau_{90}$ & Relaxation time for $90^{\circ}$ switching $(\mathrm{s})$ \\
\hline$\tau_{180}$ & Relaxation time for $180^{\circ}$ switching (s) \\
\hline$\chi_{e}$ & Electric susceptibility (Unitless) \\
\hline$\chi_{\alpha}^{\sigma}$ & Ferroelectric susceptibility of $\alpha$-variant at constant stress $(\mathrm{F} / \mathrm{m}=\mathrm{C} / \mathrm{Vm})$ \\
\hline$\psi_{\alpha}$ & Helmholtz energy density of $\alpha$-variant $\left(\mathrm{CV} / \mathrm{m}^{3}\right)$ \\
\hline
\end{tabular}

\section{Introduction}

Ferroelectric materials, such as lead zirconate titanate (PZT), lanthanum-doped lead zirconate titanate (PLZT), lead manganese niobate (PMN), polyvinylidene flouride (PVDF), and barium titanate $\left(\mathrm{BaTiO}_{3}\right)$, are being widely considered as actuators, sensors, and structural units for a range of aerospace, aeronautic, automotive, industrial and biomedical applications. Their advantages derive from the intrinsic properties of the materials. The complementary direct and converse piezoelectric effects imbue the materials with multiple design properties including sensor, actuator, self-monitoring and nondestructive evaluation, and energy harvesting capabilities. Their functionality is augmented by their solid state nature which promotes miniaturization and simplified design and reduces power requirements and heat generated by the units. Furthermore, ferroelectric actuators can provide nanometer positioning resolution and can operate at frequencies ranging from $\mathrm{DC}$ to $\mathrm{MHz}$. As detailed in the companion paper [19], ferroelectric transducers are being considered, or are already being employed, for a large number of applications including high speed valves for fuel injectors, ferroelectric memory technologies (e.g., FeRAM), nanopositioning units (e.g., AFM and STM stages), 
high speed camera shutters and autofocusing units, ultrasonic transducers for biomedical imaging and treatment, micro and pico air vehicle design, and energy harvesting devices.

However, the ferroelectric properties that imbue the materials with unique transducer capabilities also produce rate-dependent hysteresis, creep, and constitutive nonlinearities as illustrated in Figure 1. The field-polarization and field-strain data from [26] illustrates that rate-dependent effects are significant at frequencies as low as $1 \mathrm{~Hz}$. PZT data from [24] illustrates that for fixed field inputs, both the strain and polarization exhibit significant creep on timescales of 1 to 20 seconds. Finally, the MFC data from [8] illustrates nested minor loop behavior typical of moderate drive regimes. Whereas the full switching behavior shown in Figure 1(a)-(e) will typically not be encountered in applications, general models must account for the full range of rate-dependent and creep behavior to provide comprehensive device characterization.

To be optimally utilized in applications, constitutive models must satisfy the following criteria: (i) they must adequately quantify the range of rate-dependent behavior exhibited by the materials, (ii) they must be in a form that can be readily extended to provided distributed models, for complex structures and devices, that are amenable to finite element implementation, (iii) they must be efficient to implement, (iv) they must be readily calibrated for a specific material or device, and (v) models employed in control designs can prove advantageous if they admit the construction of approximate inverse relations.

It is shown in [18-21] that the homogenized energy model (HEM) satisfies (i) and (ii). Furthermore, the construction of approximate inverses for previous formulations of the framework are reported in [4,18] and inversion techniques for the present formulation [19] are addressed in [15]. In this paper, we address (iii) and (iv) by presenting highly efficient implementation techniques and data-driven parameter estimation algorithms for the homogenized energy model. In combination, this

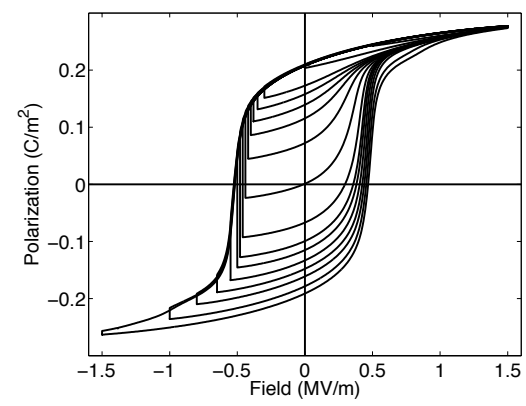

(a)

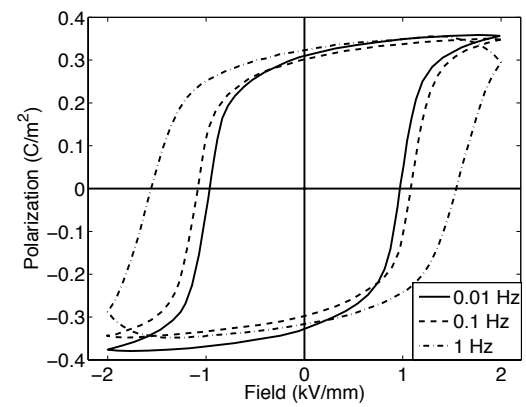

(d)

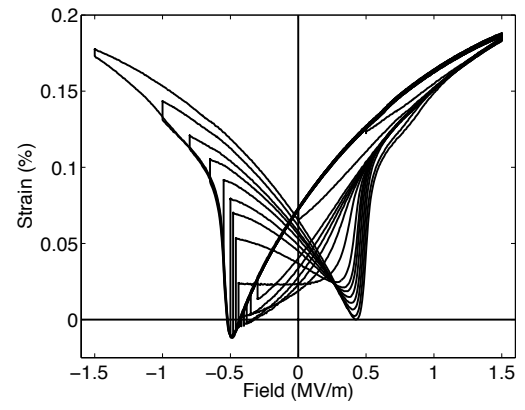

(b)

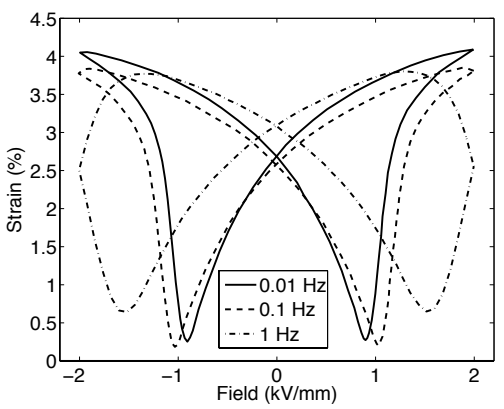

(e)

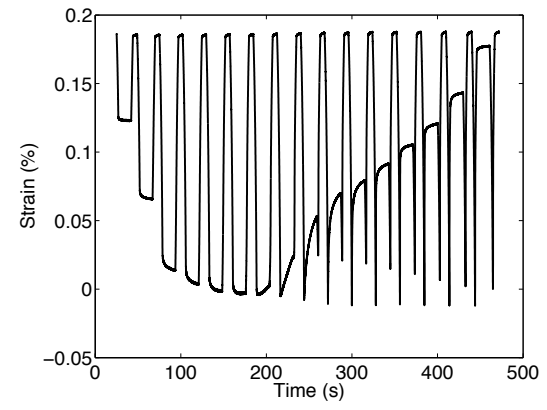

(c)

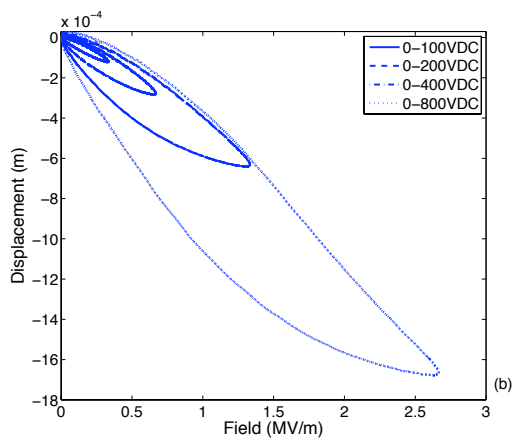

(f)

Figure 1: (a) Field-polarization, (b) field-strain and (c) time-strain PZT data from [24]. Ratedependent (d) polarization and (e) strain PZT data from [26]. (f) Field-strain MFC data from [8]. 
provides the framework with significant flexibility for use in simulation packages, design algorithms, and control designs for systems utilizing ferroelectric transducers.

To provide context for the framework, we first summarize the mechanisms that produce the hysteretic behavior shown in Figure 1. The creep and rate-dependencies are due to the fact that kinetics associated with dipole switching typically differ from mechanical or electrical loading rates. Furthermore, it is discussed in [19] that the $180^{\circ}$ switching associated with large polarization changes often occurs at a different rate than $90^{\circ}$ switching associated with large strain changes. In combination, this establishes that multiple time scales must be incorporated in dynamic models.

As illustrated for a PZT-based macro-fiber composite (MFC) in Figure 2, hysteresis also involves multiple spatial scales. Within a domain, strains or changes in polarization are due to stress-induced material deformations or field-induced ion movement and the behavior is often reversible and linear. Field or stress-induced dipole switching at the grain level produces irreversible hysteresis in both the field-polarization and field-strain relations. For single crystal materials comprised of a single grain, the switching is typically rapid thus producing sharp hysteresis and butterfly loops in quasistatic operating regimes. For polycrystalline materials with distributed interaction and coercive fields, hysteresis and butterfly loops are smoothed due to nonuniform grain contributions. The behavior of hysteresis loops is further modified when hysteretic actuator or sensor materials are employed on distributed structures.

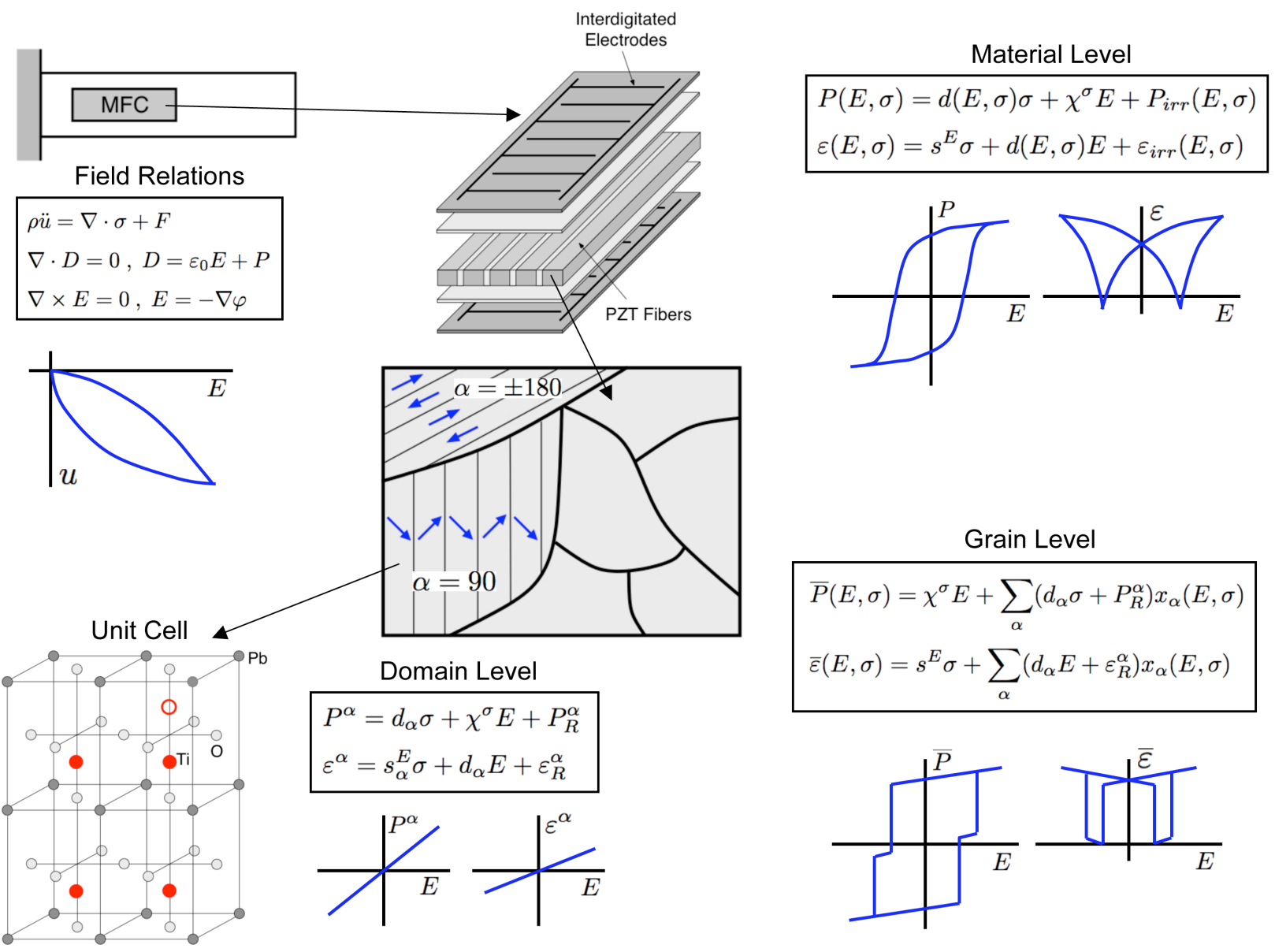

Figure 2: Multiscale behavior of a PZT-based MFC transducer at the application, device, material and unit cell levels. 
As noted in Figure 2, material-level constitutive relations for the polarization $P$ and strain $\varepsilon$ can generically be expressed as

$$
\begin{aligned}
& P(E, \sigma)=d(E, \sigma) \sigma+\chi^{\sigma} E+P_{i r r}(E, \sigma) \\
& \varepsilon(E, \sigma)=s^{E} \sigma+d(E, \sigma) E+\varepsilon_{i r r}(E, \sigma)
\end{aligned}
$$

where $E, \sigma$ are input fields and stresses, $\chi^{\sigma}$ is the ferroelectric susceptibility at constant stress, $s^{E}$ is the elastic compliance at constant field, and $d$ is a piezoelectric coupling coefficient. We note that $d(E, \sigma), P_{i r r}(E, \sigma)$ and $\varepsilon_{i r r}(E, \sigma)$ incorporate the nonlinear and irreversible history-dependence due to dipole switching.

Various modeling hierarchies can be defined by the manner in which $d, P_{i r r}$ and $\varepsilon_{i r r}$ are constructed. Micromechanical, or microscopically-motivated models are based on an energy description of the material at the domain or grain level in combination with various homogenization techniques to provide expressions for the nonlinear, effective components $d, P_{i r r}$ and $\varepsilon_{i r r}$. Phenomenological models circumvent the difficulties associated with quantifying complex, or poorly understood, micro-scale physics by constructing relations for $d, P_{i r r}$ and $\varepsilon_{i r r}$ based on macroscale observations or experimental measurements, often guided by thermodynamic constraints. Details regarding various models for ferroelectric materials can be found in $[2,9,13,16,18,19]$.

The homogenized energy model is a multiscale, micromechanical approach that begins with energy analysis at the domain level to construct the linear relations shown in Figure 2 for $P^{\alpha}$ and $\varepsilon^{\alpha}$ where $\alpha$ designates the dipole variant; e.g., $\alpha= \pm 180,90$ for tetragonal materials. Switching processes due to domain wall nucleation and movement are incorporated by tracking the evolution of dipole fractions $x_{\alpha}$ which serve as internal variables. Differential equations quantifying the dynamics of $x_{\alpha}$ are driven by likelihood rates constructed using Boltzmann theory to quantify the scaled probability of transitioning between stable equilibria associated with dipole variants. This incorporates the rate-dependence and multiple-time scales exhibited by the data in Figure 1 and yields grain-level or single crystal kernels $\bar{P}$ and $\bar{\varepsilon}$. For polycrystalline materials, material-level models are constructed by assuming that properties such as coercive fields, critical driving forces, and interaction fields are manifestations of underlying densities rather than constants.

The three primary issues that must be addressed to construct implementation algorithms are: (i) efficient approximation of the integrals, posed on infinite and semi-infinite domains, arising in the homogenization step used to construct the irreversible components $d(E, \sigma), P_{\text {irr }}(E, \sigma)$ and $\varepsilon_{\text {irr }}(E, \sigma)$, (ii) efficient approximation of the evolution equations for $x_{\alpha}$, and (iii) efficient evaluation of the likelihoods which incorporate rate-dependent effects and drive the evolution equations. The first issue is addressed by employing density representations that admit efficient numerical approximation on finite domains whereas implicit Euler discretizations readily address the second issue. The repeated evaluation of likelihoods is avoided by constructing arrays that permit high-speed access during model implementation. In combination, the evaluation of $d(E, \sigma), P_{i r r}(E, \sigma)$ and $\varepsilon_{i r r}(E, \sigma)$ are reduced to operations solely involving componentwise matrix multiplication and summation. Hence the operations are highly efficient and inherently parallelizable which facilitates subsequent implementation using devices such as field programmable gate arrays (FPGA) [3].

We note that approximate model inversion techniques rely on forward solutions of the model. Hence the efficiency of forward algorithms also directly contributes to the speed of inverse algorithms.

There are two primary issues that must be addressed when employing gradient-based optimization algorithms for the parameter estimation required for model calibration: (i) determination of accurate initial parameter estimates and (ii) efficient and accurate construction of gradients or Jacobians. Due to its physical nature, parameters in the homogenized energy model can be directly correlated with properties of measured data. We utilize that here to construct data-driven algorithms to determine 
initial parameter estimates. Secondly, we construct analytic Jacobian relations which significantly improves both the speed and accuracy of optimization routines. In combination, this helps mitigate some of the difficulties associated with the shallow slopes and nonconvexity (multiple local minima) inherent to functionals employed in parameter estimation problems of this type.

We note that various aspects of the implementation and data-driven parameter estimation algorithms can be optimized to provide improved performance for specific data sets. Instead, our goal was to provide algorithms that balance simplicity, robustness, accuracy, and optimality for a wide range of materials, applications and operating regimes. Points were algorithms can be modified or optimized are noted in the discussion.

Following a brief summary of the model in Section 2, highly efficient implementation algorithms are presented in Section 3. The parameter estimation problem is discussed in Section 4 where we provide algorithms to construct analytic Jacobians and data-driven techniques to determine initial parameter estimates. Model calibration and validation are illustrated in Section 5 using PZT data from [24] and single crystal $\mathrm{BaTiO}_{3}$ data from [5]. Additional examples illustrating the capability of the model to characterize creep and rate-dependent data, such as the shown in Figure 1, are provided in the companion paper [19] which details the model development.

\section{Polarization and Strain Models}

As detailed in [19], consideration of $180^{\circ}$ dipole switching yields a macroscopic model quantifying the nonlinear and hysteretic field-polarization map for ferroelectric materials whereas the additional incorporation of $90^{\circ}$ switching yields a homogenized model quantifying both the polarization and strains due to input fields and stresses. We summarize the latter model and indicate simplifications that occur if considering only the polarization in the absence of applied stresses.

\section{Polarization and Strain Model}

At the lattice level, we consider the Helmholtz and Gibbs energy densities

$$
\psi_{\alpha}(P, \varepsilon)=\frac{1}{2} \eta_{\alpha}^{\varepsilon}\left(P-P_{R}^{\alpha}\right)^{2}+\frac{1}{2} Y_{\alpha}^{P}\left(\varepsilon-\varepsilon_{R}^{\alpha}\right)^{2}+h_{\alpha}\left(P-P_{R}^{\alpha}\right)\left(\varepsilon-\varepsilon_{R}^{\alpha}\right)
$$

and

$$
G_{\alpha}(E, \sigma ; P, \varepsilon)=\psi_{\alpha}(P, \varepsilon)-E P-\sigma \varepsilon
$$

where we indicate $\pm 180^{\circ}$ and $90^{\circ}$ orientations by $\alpha= \pm, 90$. As summarized in the nomenclature table at the beginning of the paper, $P_{R}^{\alpha}, \varepsilon_{R}^{\alpha}, \eta_{\alpha}^{\varepsilon}, Y_{\alpha}^{P}$ and $h_{\alpha}$ denote the remanence polarization, remanence strain, inverse susceptibility at constant strain, elastic stiffness at constant polarization, and piezoelectric constant.

For a fixed applied field and stress, the conditions $\frac{\partial G}{\partial P}=0$ and $\frac{\partial G}{\partial \varepsilon}=0$ yield the relations

$$
\begin{aligned}
& P_{m}^{\alpha}=P_{R}^{\alpha}+\chi_{\alpha}^{\sigma} E+d_{\alpha} \sigma \\
& \varepsilon_{m}^{\alpha}=\varepsilon_{R}^{\alpha}+d_{\alpha} E+s_{\alpha}^{E} \sigma
\end{aligned}
$$

where

$$
\chi_{\alpha}^{\sigma}=\frac{Y_{\alpha}^{P}}{Y_{\alpha}^{P} \eta_{\alpha}^{\varepsilon}-h_{\alpha}^{2}}, d_{\alpha}=\frac{h_{\alpha}}{h_{\alpha}^{2}-Y_{\alpha}^{P} \eta_{\alpha}^{\varepsilon}}, s_{\alpha}^{E}=\frac{\eta_{\alpha}^{\varepsilon}}{Y_{\alpha}^{P} \eta_{\alpha}^{\varepsilon}-h_{\alpha}^{2}}
$$

are the ferroelectric susceptibility at constant stress, the piezoelectric constant, and elastic compliance at constant field. The minimum of the Gibbs energy in each $\alpha$-well can then be expressed as

$$
G_{\alpha m}(E, \sigma)=-\frac{1}{2} \chi_{\alpha}^{\sigma} E^{2}-\frac{1}{2} s_{\alpha}^{E} \sigma^{2}-d_{\alpha} E \sigma-E P_{R}^{\alpha}-\sigma \varepsilon_{R}^{\alpha}
$$


The dipole fractions $x_{+}, x_{-}$and $x_{90}$ associated with positively, negatively, and $90^{\circ}$ degree dipoles evolve according to the differential equations

$$
\begin{aligned}
& \dot{x}_{-}=-\left(p_{-90}+p_{-+}\right) x_{-}+p_{90-} x_{90}+p_{+-} x_{+} \\
& \dot{x}_{90}=p_{-90} x_{-}-\left(p_{90-}+p_{90+}\right) x_{90}+p_{+90} x_{+} \\
& \dot{x}_{+}=p_{-+} x_{-}+p_{90+} x_{90}-\left(p_{+90}+p_{+-}\right) x_{+}
\end{aligned}
$$

where

$$
p_{\alpha \beta}(E, \sigma)=\frac{1}{\tau_{\alpha \beta}} e^{-\Delta G_{\alpha \beta}^{a}(E, \sigma) V / k T}
$$

quantifies the likelihood of transitioning from an $\alpha$-well to a $\beta$-well. As noted in Table 1, (2) can be simplified using the relation $x_{+}+x_{-}+x_{90}=1$. The activation energy is specified by the relation

$$
\Delta G_{\alpha \beta}^{a}\left(E, \sigma ; F_{c}\right)=\left\{\begin{array}{cc}
\Delta G_{0}\left(1-F_{\alpha \beta}(E, \sigma) / F_{c}\right)^{2} & , \quad F_{\alpha \beta}(E, \sigma) \leq F_{c} \\
0 & , \quad F_{\alpha \beta}(E, \sigma)>F_{c} .
\end{array}\right.
$$

Here $F_{\alpha \beta}(E, \sigma)=G_{\alpha m}(E, \sigma)-G_{\beta m}(E, \sigma)$ is the thermodynamic driving force. The specific form of $F_{\alpha \beta}$, based on the physical assumption that

$$
\begin{aligned}
& \chi_{+}^{\sigma}=\chi_{-}^{\sigma}=\chi_{90}^{\sigma}=\chi^{\sigma} \\
& s_{+}^{E}=s_{-}^{E}=s_{90}^{E}=s^{E} \\
& P_{R}^{90}=0, P_{R}^{+}=-P_{R}^{-}, \varepsilon_{R}^{+}=\varepsilon_{R}^{-} \\
& d_{90}=0, d_{-}=-d_{+} \\
& \tau_{90-}=\tau_{-90}=\tau_{90+}=\tau_{+90}=\tau_{90}, \tau_{+-}=\tau_{-+}=\tau_{180},
\end{aligned}
$$

is given in Table 1 . Note that

$$
\Delta G_{0}=\left\{\begin{array}{cl}
\frac{1}{4} F_{c} & , 180^{\circ} \text { Switching } \\
\frac{1}{16} F_{c} & , \quad 90^{\circ} \text { Switching }
\end{array}\right.
$$

is the energy barrier at zero driving force.

The polarization and strain kernels are given by

$$
\bar{P}=\sum_{\alpha= \pm, 90} x_{\alpha} P_{m}^{\alpha} \quad, \quad \bar{\varepsilon}=\sum_{\alpha= \pm, 90} x_{\alpha} \varepsilon_{m}^{\alpha}
$$

Based on (1) and (4), these relations can be expressed as

$$
\begin{aligned}
& \bar{P}(E, \sigma)=\bar{d}(E, \sigma) \sigma+\chi^{\sigma} E+\bar{P}_{i r r}(E, \sigma) \\
& \bar{\varepsilon}(E, \sigma)=s^{E} \sigma+\bar{d}(E, \sigma) E+\bar{\varepsilon}_{i r r}(E, \sigma)
\end{aligned}
$$

where

$$
\begin{aligned}
& \bar{d}(E, \sigma)=\sum_{\alpha= \pm, 90} d_{\alpha} x_{\alpha}(E, \sigma) \\
& \bar{P}_{i r r}(E, \sigma)=\sum_{\alpha= \pm, 90} P_{R}^{\alpha} x_{\alpha}(E, \sigma) \\
& \bar{\varepsilon}_{i r r}(E, \sigma)=\sum_{\alpha= \pm, 90} \varepsilon_{R}^{\alpha} x_{\alpha}(E, \sigma) .
\end{aligned}
$$


In the final step in the development of [19], macroscopic models

$$
\begin{aligned}
& P\left(E(t), \sigma(t) ; x_{+}^{0}\right)=\int_{0}^{\infty} \int_{-\infty}^{\infty} \bar{P}\left(E(t)+E_{I}, \sigma(t) ; F_{c}\right) \nu_{I}\left(E_{I}\right) \nu_{c}\left(F_{c}\right) d E_{I} d F_{c} \\
& \varepsilon\left(E(t), \sigma(t) ; x_{+}^{0}\right)=\int_{0}^{\infty} \int_{-\infty}^{\infty} \bar{\varepsilon}\left(E(t)+E_{I}, \sigma(t) ; F_{c}\right) \nu_{I}\left(E_{I}\right) \nu_{c}\left(F_{c}\right) d E_{I} d F_{c}
\end{aligned}
$$

are constructed by considering interaction fields $E_{I}$ and thermodynamic driving forces $F_{c}$ to be manifestations of underlying densities $\nu_{I}$ and $\nu_{c}$. The final models can thus be expressed as

$$
\begin{aligned}
& P(E, \sigma)=d(E, \sigma) \sigma+\chi^{\sigma} E+P_{i r r}(E, \sigma) \\
& \varepsilon(E, \sigma)=s^{E} \sigma+d(E, \sigma) E+\varepsilon_{i r r}(E, \sigma)
\end{aligned}
$$

where

$$
\begin{aligned}
& d(E, \sigma)=\int_{0}^{\infty} \int_{-\infty}^{\infty} \bar{d}\left(E_{e} ; F_{c}\right) \nu_{I}\left(E_{I}\right) \nu_{c}\left(F_{c}\right) d E_{I} d F_{c} \\
& P_{i r r}(E, \sigma)=\int_{0}^{\infty} \int_{-\infty}^{\infty} \bar{P}_{i r r}\left(E_{e} ; F_{c}\right) \nu_{I}\left(E_{I}\right) \nu_{c}\left(F_{c}\right) d E_{I} d F_{c} \\
& \varepsilon_{i r r}(E, \sigma)=\int_{0}^{\infty} \int_{-\infty}^{\infty} \bar{\varepsilon}_{i r r}\left(E_{e} ; F_{c}\right) \nu_{I}\left(E_{I}\right) \nu_{c}\left(F_{c}\right) d E_{I} d F_{c}
\end{aligned}
$$

Here $E_{e}(t)=E(t)+E_{I}$ is the effective field.

Details regarding various choices for the densities are provided in [19]. For the implementation and parameter estimation algorithms detailed in this paper, we employ the representations

$$
\begin{array}{lll}
\nu_{c}\left(F_{c}\right) & =\frac{1}{\sum_{\ell} \alpha_{\ell}} \sum_{k=k_{\alpha}}^{K_{\alpha}} \alpha_{k} \phi_{k}\left(F_{c}\right) \quad, \quad \phi_{k}\left(F_{c}\right)=\frac{1}{\sigma_{c}^{k} F_{c} \sqrt{2 \pi}} e^{-\left[\ln \left(F_{c}\right)-\ln \left(\bar{F}_{c}\right)\right]^{2} / 2\left(\sigma_{c}^{k}\right)^{2}} \quad, \quad \sigma_{c}^{k}=2^{k} \sigma_{c} \\
\nu_{I}\left(E_{I}\right) & =\frac{1}{\sum_{\ell} \beta_{\ell}} \sum_{k=k_{\beta}}^{K_{\beta}} \beta_{k} \varphi_{k}\left(E_{I}\right) \quad, \quad \varphi_{k}\left(E_{I}\right)=\frac{1}{\sigma_{I}^{k} \sqrt{2 \pi}} e^{-E_{I}^{2} / 2\left(\sigma_{I}^{k}\right)^{2}} & , \sigma_{I}^{k}=2^{k} \sigma_{I}
\end{array}
$$

where the preceding sums ensure integration to unity. During model calibration, the parameters $\left\{\alpha_{k}, \beta_{k}\right\}$ are determined through a least squares fit to the data.

The complete polarization and strain model is summarized in Table 1.

\section{Parameters for the Polarization-Strain Model}

To construct the density basis functions $\varphi_{k}\left(E_{I}\right)$ and $\phi_{k}\left(F_{c}\right)$, it is necessary to determine values for the driving force mean $\mu_{c}=\ln \left(\bar{F}_{c}\right)$ and variance $\sigma_{c}^{2}$ as well as the interaction field variance $\sigma_{I}^{2}$. We denote this set of parameters by

$$
\bar{p}=\left[\mu_{c}, \sigma_{c}^{2}, \sigma_{I}^{2}\right] .
$$

We provide an algorithm in Section 4.2 to estimate values for these parameters based on measured attributes of $E-P$ data. We note that these parameters are not updated or optimized through a least squares fit to data.

Based on the assumption (4), the remaining parameters are denoted by

$$
p=\left[P_{R}^{+}, \varepsilon_{R}^{+}, \varepsilon_{R}^{90}, \chi^{\sigma}, d_{+}, s^{E}, \gamma, \tau_{90}, \tau_{180}, \alpha_{k}, \beta_{k}\right] .
$$

This comprises the set that is optimized during model calibration. 


\section{Homogenized Energy Model:}

$$
\begin{aligned}
& P(E, \sigma)=d(E, \sigma) \sigma+\chi^{\sigma} E+P_{i r r}(E, \sigma) \\
& \varepsilon(E, \sigma)=s^{E} \sigma+d(E, \sigma) E+\varepsilon_{i r r}(E, \sigma)
\end{aligned}
$$

where

$$
\begin{aligned}
& d(E, \sigma)=\int_{0}^{\infty} \int_{-\infty}^{\infty} \bar{d}\left(E+E_{I} ; F_{c}\right) \nu_{I}\left(E_{I}\right) \nu_{c}\left(F_{c}\right) d E_{I} d F_{c} \\
& P_{i r r}(E, \sigma)=\int_{0}^{\infty} \int_{-\infty}^{\infty} \bar{P}_{i r r}\left(E+E_{I} ; F_{c}\right) \nu_{I}\left(E_{I}\right) \nu_{c}\left(F_{c}\right) d E_{I} d F_{c} \\
& \varepsilon_{i r r}(E, \sigma)=\int_{0}^{\infty} \int_{-\infty}^{\infty} \bar{\varepsilon}_{i r r}\left(E+E_{I} ; F_{c}\right) \nu_{I}\left(E_{I}\right) \nu_{c}\left(F_{c}\right) d E_{I} d F_{c}
\end{aligned}
$$

Kernels:

$$
\begin{aligned}
& \bar{d}\left(E, \sigma ; F_{c}\right)=\sum_{\alpha= \pm, 90} d_{\alpha} x_{\alpha}\left(E ; F_{c}\right) \\
& \bar{P}_{i r r}\left(E, \sigma ; F_{c}\right)=\sum_{\alpha= \pm, 90} P_{R}^{\alpha} x_{\alpha}\left(E ; F_{c}\right) \\
& \bar{\varepsilon}_{i r r}\left(E, \sigma ; F_{c}\right)=\sum_{\alpha= \pm, 90} \varepsilon_{R}^{\alpha} x_{\alpha}\left(E ; F_{c}\right)
\end{aligned}
$$

Evolution Equations: $\left(t ; E, \sigma, F_{c}\right)$

$$
\begin{aligned}
& \dot{x}_{-}=-\left(p_{-90}+p_{-+}+p_{90-}\right) x_{-}+\left(p_{+-}-p_{90-}\right) x_{+}+p_{90-} \\
& \dot{x}_{+}=\left(p_{-+}-p_{90+}\right) x_{-}-\left(p_{+90}+p_{+-}+p_{90+}\right) x_{+}+p_{90+} \\
& x_{90}=1-x_{+}-x_{-}
\end{aligned}
$$

Likelihoods: $\alpha, \beta= \pm 90$

$$
p_{\alpha \beta}\left(E, \sigma ; F_{c}\right)=\frac{1}{\tau_{\alpha \beta}} e^{-\gamma \Delta G_{\alpha \beta}^{a}\left(E, \sigma ; F_{c}\right)}, \gamma=V / k T
$$

Activation Energy: $\alpha, \beta= \pm 90$

$$
\begin{aligned}
& \Delta G_{\alpha \beta}^{a}\left(E, \sigma ; F_{c}\right)=\left\{\begin{array}{cc}
\Delta G_{0}\left(1-F_{\alpha \beta}(E, \sigma) / F_{c}\right)^{2} & , \quad F_{\alpha \beta}(E, \sigma) \leq F_{c} \\
0 & , \quad F_{\alpha \beta}(E, \sigma)>F_{c}
\end{array}\right. \\
& F_{90+}(E, \sigma)=d_{+} E \sigma+P_{R}^{+} E-\left(\varepsilon_{R}^{90}-\varepsilon_{R}^{+}\right) \sigma \quad, \quad F_{+90}=-F_{90+} \\
& F_{90-}(E, \sigma)=-d_{+} E \sigma-P_{R}^{+} E-\left(\varepsilon_{R}^{90}-\varepsilon_{R}^{+}\right) \sigma \quad, \quad F_{-90}=-F_{90-} \\
& F_{+-}(E, \sigma)=-2 d_{+} E \sigma-2 E P_{R}^{+} \quad, \quad F_{-+}=-F_{+-}
\end{aligned}
$$

Densities:

$$
\begin{array}{ll}
\nu_{c}\left(F_{c}\right)=\frac{1}{\sum_{\ell} \alpha_{\ell}} \sum_{k=k_{\alpha}}^{K_{\alpha}} \alpha_{k} \phi_{k}\left(F_{c}\right) & , \quad \phi_{k}\left(F_{c}\right)=\frac{1}{\sigma_{c}^{k} F_{c} \sqrt{2 \pi}} e^{-\left[\ln \left(F_{c}\right)-\ln \left(\bar{F}_{c}\right)\right]^{2} / 2\left(\sigma_{c}^{k}\right)^{2}} \\
\nu_{I}\left(E_{I}\right)=\frac{1}{\sum_{\ell} \beta_{\ell}} \sum_{k=k_{\beta}}^{K_{\beta}} \beta_{k} \varphi_{k}\left(E_{I}\right) \quad, \quad \varphi_{k}\left(E_{I}\right)=\frac{1}{\sigma_{I}^{k} \sqrt{2 \pi}} e^{-E_{I}^{2} / 2\left(\sigma_{I}^{k}\right)^{2}}
\end{array}
$$

Table 1: Components of the polarization and strain model based on $90^{\circ}$ dipole switching. 


\section{Polarization Model}

If solely quantifying the polarization in the absence of applied stresses, one can consider energy landscapes and dipole fractions associated only with positively and negatively oriented dipoles which we designate by $\alpha= \pm$. It is shown in [19] that a complete characterization of the Helmholtz and Gibbs energy densities yields likelihoods $p_{-+}$and $p_{+-}$formulated in terms of complementary error functions whereas formulation in terms of the activation energy yields likelihood relations analogous to (3). We focus on this latter case and note that analogous implementation and parameter estimation algorithms can be constructed for the error function formulation.

The polarization model based on $180^{\circ}$ dipole switching is summarized in Table 2.

\section{Polarization Model Parameters}

In this case, we employ a coercive field density $\nu_{c}\left(E_{c}\right)$ having the same form as the driving force density $\nu_{c}\left(F_{c}\right)$. Hence it is again necessary to prescribe data-driven algorithms to determine values of the coefficients $\bar{p}$ in (6). The parameters to be estimated through a least squares fit to data are

$$
p=\left[\eta, P_{R}, \gamma, \tau, \alpha_{k}, \beta_{k}\right]
$$

We discuss associated optimization algorithms in Section 4 and data-driven techniques to obtain initial parameter estimates in Section 4.2.

\section{Implementation Algorithms}

To facilitate implementation of the models summarized in Tables 1 and 2 , or their inverses, three issues must be addressed: (i) approximation of the infinite-domain integrals in a manner that retains its accuracy for the basis expansions (5), (ii) evaluations of the likelihoods $p_{\alpha \beta}$ in an efficient manner, and (iii) efficient solution of the differential equations (11) and (19).

\subsection{Quadrature Techniques}

To implement the model, the integrals defining the terms $d, P_{i r r}$ and $\varepsilon_{i r r}$ in (9) and (17) must be approximated in a manner that is both efficient and accurate. We first note that by employing the relations (10), these terms can be expressed as

$$
\begin{aligned}
& d(E, \sigma)=\sum_{\alpha= \pm, 90} d_{\alpha} \int_{0}^{\infty} \int_{-\infty}^{\infty} x_{\alpha}\left(E_{e} ; F_{c}\right) \nu_{I}\left(E_{I}\right) \nu_{c}\left(F_{c}\right) d E_{I} d F_{c} \\
& P_{i r r}(E, \sigma)=\sum_{\alpha= \pm, 90} P_{R}^{\alpha} \int_{0}^{\infty} \int_{-\infty}^{\infty} x_{\alpha}\left(E_{e} ; F_{c}\right) \nu_{I}\left(E_{I}\right) \nu_{c}\left(F_{c}\right) d E_{I} d F_{c} \\
& \varepsilon_{i r r}(E, \sigma)=\sum_{\alpha= \pm, 90} \varepsilon_{R}^{\alpha} \int_{0}^{\infty} \int_{-\infty}^{\infty} x_{\alpha}\left(E_{e} ; F_{c}\right) \nu_{I}\left(E_{I}\right) \nu_{c}\left(F_{c}\right) d E_{I} d F_{c} .
\end{aligned}
$$

Secondly, it is noted in [19] that the densities satisfy exponential decay constraints. Hence approximation algorithms can be defined on finite domains rather than necessitating quadrature techniques for infinite and semi-infinite domains. We illustrate here a trapezoidal formula and refer the reader to [18] for details regarding composite Gaussian quadrature techniques. We note that truncation of the domains and use of composite techniques is more efficient to implement than Gauss-Hermite and Gauss-Laguerre algorithms, that are designed specifically for infinite and semi-infinite intervals, 
$\underline{\text { Homogenized Energy Model: }}$

$$
P(E)=\frac{E}{\eta}+P_{i r r}
$$

where

$$
P_{i r r}(E)=\int_{0}^{\infty} \int_{-\infty}^{\infty} \bar{P}_{i r r}\left(E+E_{I} ; F_{c}\right) \nu_{I}\left(E_{I}\right) \nu_{c}\left(F_{c}\right) d E_{I} d F_{c}
$$

Kernel:

$$
\bar{P}_{\text {irr }}\left(E ; F_{c}\right)=\sum_{\alpha= \pm 90} P_{R}^{\alpha} x_{\alpha}\left(E ; F_{c}\right)=-P_{R}+2 P_{R} x_{+}
$$

Evolution Equations: $\left(t, E ; F_{c}\right)$

$$
\begin{aligned}
& \dot{x}_{+}=-\left(p_{-+}+p_{+-}\right) x_{+}+p_{-+} \\
& x_{-}=1-x_{+}
\end{aligned}
$$

$\underline{\text { Likelihoods }}$ (Choice 1): $\alpha, \beta= \pm$

$$
p_{\alpha \beta}\left(E ; F_{c}\right)=\frac{1}{\tau} e^{-\gamma \Delta G_{\alpha \beta}^{a}\left(E ; F_{c}\right)}, \gamma=V / k T
$$

Activation Energy: $\alpha, \beta= \pm 90, F_{c}=2 E_{c} P_{R}, \Delta G_{0}=\frac{F_{c}}{4}$

$$
\begin{gathered}
\Delta G_{\alpha \beta}^{a}\left(E ; F_{c}\right)=\left\{\begin{array}{cr}
\Delta G_{0}\left(1-F_{\alpha \beta}(E) / F_{c}\right)^{2} & , \quad F_{\alpha \beta}(E) \leq F_{c} \\
0 \quad & , \quad F_{\alpha \beta}(E)>F_{c}
\end{array}\right. \\
F_{-+}(E)=2 E P_{R} \quad, \quad F_{+-}=-F_{-+}
\end{gathered}
$$

Likelihoods (Choice 2):

$$
p_{+-}\left(E ; E_{c}\right)=\frac{\gamma_{1}}{\operatorname{erfcx}\left[\gamma_{2}\left(-E-E_{c}\right)\right]}, p_{-+}\left(E ; E_{c}\right)=\frac{\gamma_{1}}{\operatorname{erfcx}\left[\gamma_{2}\left(E-E_{c}\right)\right]}
$$

where

$$
\gamma_{1}=\frac{1}{\tau} \sqrt{\frac{2 V \eta}{\pi k T}} \quad, \quad \gamma_{2}=\sqrt{\frac{V}{2 k T \eta}}
$$

Densities:

$$
\begin{array}{ll}
\nu_{c}\left(F_{c}\right)=\frac{1}{\sum_{\ell} \alpha_{\ell}} \sum_{k=k_{\alpha}}^{K_{\alpha}} \alpha_{k} \phi_{k}\left(F_{c}\right) \quad, \quad \phi_{k}\left(F_{c}\right)=\frac{1}{\sigma_{c}^{k} F_{c} \sqrt{2 \pi}} e^{-\left[\ln \left(F_{c}\right)-\ln \left(\bar{F}_{c}\right)\right]^{2} / 2\left(\sigma_{c}^{k}\right)^{2}} \\
\nu_{I}\left(E_{I}\right)=\frac{1}{\sum_{\ell} \beta_{\ell}} \sum_{k=k_{\beta}}^{K_{\beta}} \beta_{k} \varphi_{k}\left(E_{I}\right) \quad, \quad \varphi_{k}\left(E_{I}\right)=\frac{1}{\sigma_{I}^{k} \sqrt{2 \pi}} e^{-E_{I}^{2} / 2\left(\sigma_{I}^{k}\right)^{2}}
\end{array}
$$

Table 2: Components of the polarization model based on $180^{\circ}$ dipole switching and the physical assumptions (4) with $P_{R} \equiv P_{R}^{+}$and $\tau \equiv \tau_{180}$. 
because they allow accurate evaluation of the density expansions (5) and construction of lookup tables to improve algorithm efficiency as detailed in Section 3.2.

If we let $F_{c_{i}}, E_{I_{j}}$ and $v_{i}, w_{j}$ respectively denote the quadrature points and weights, we obtain the approximate relations

$$
\int_{0}^{\infty} \int_{-\infty}^{\infty} x_{\alpha}\left(E+E_{I} ; F_{c}\right) \nu_{I}\left(E_{I}\right) \nu_{c}\left(F_{c}\right) d E_{I} d F_{c} \approx \sum_{i=1}^{N_{i}} \sum_{j=1}^{N_{j}} x_{\alpha}\left(E+E_{I_{j}} ; F_{c_{i}}\right) \nu_{I}\left(E_{I_{j}}\right) \nu_{c}\left(F_{c_{j}}\right) v_{i} w_{j} .
$$

For the validation results reported in Section 5, sufficient accuracy was achieved using a trapezoid rule with $N_{i}=N_{j}=41$.

\subsection{Determination of Phase Fractions and Likelihoods}

From (24), it is observed that evaluation of the model requires the solution of $N_{i} \times N_{j}$ differential equations for each phase fraction $x_{\alpha}$ which in turn requires $N_{i} \times N_{j}$ evaluations of the likelihoods $p_{\alpha \beta}$. Due to its relative simplicity, we first illustrate a highly efficient implementation algorithm for the $180^{\circ}$ polarization model. In Section 3.2 .2 , an analogous, but slightly more complicated, algorithm is developed for the $90^{\circ}$ polarization-strain model.

\subsubsection{Polarization Model}

To approximate the solution of (19), it is shown in [4] that for sufficiently small stepsizes $\Delta t$, an implicit Euler discretization yields the stable difference relation

$$
x_{+}^{k+1}=c_{1}^{k+1} x_{+}^{k}+c_{2}^{k+1}
$$

where $t_{k}=k \Delta t, E_{e}^{k}=E_{e}\left(t_{k}\right)=E\left(t_{k}\right)+E_{I}, x_{+}^{k}=x_{+}\left(t_{k} ; e_{E}^{k}, F_{c}\right)$ and

$$
\begin{aligned}
c_{1}^{k+1} & =\frac{1}{1+\Delta t\left[p_{-+}\left(E_{e}^{k+1} ; F_{c}\right)+p_{+-}\left(E_{e}^{k+1} ; F_{c}\right)\right]} \\
c_{2}^{k+1} & =\frac{p_{-+}\left(E_{e}^{k+1} ; F_{c}\right) \Delta t}{1+\Delta t\left[p_{-+}\left(E_{e}^{k+1} ; F_{c}\right)+p_{+-}\left(E_{e}^{k+1} ; F_{c}\right)\right]} .
\end{aligned}
$$

From (16) and (24), it follows that

$$
P\left(E^{k}\right)=\frac{E^{k}}{\eta}-P_{R}+2 P_{R} V^{T} X_{+}^{k} W
$$

where the vectors

$$
\begin{aligned}
V^{T} & =\left[v_{1} \nu_{c}\left(F_{c_{1}}\right), \cdots, v_{N_{i}} \nu_{c}\left(F_{c_{N_{i}}}\right)\right]_{1 \times N_{i}} \\
W^{T} & =\left[w_{1} \nu_{I}\left(E_{I_{1}}\right), \cdots, w_{N_{j}} \nu_{I}\left(E_{I_{N_{j}}}\right)\right]_{1 \times N_{j}}
\end{aligned}
$$

incorporate the quadrature weights and densities evaluated at the quadrature points. The $N_{i} \times N_{j}$ matrix $X_{+}^{k}$ quantifies the dipole orientations at the quadrature points and is defined as

$$
\begin{aligned}
{\left[X_{+}^{k+1}\right]_{i j}=} & x_{+}\left(t_{k+1}, E^{k+1}+E_{I_{j}} ; F_{c_{i}}\right) \\
= & \frac{x_{+}\left(t_{k}, E^{k}+E_{I_{j}} ; F_{c_{i}}\right)}{1+\Delta t\left[p_{-+}\left(E^{k+1}+E_{I_{j}} ; F_{c_{j}}\right)+p_{+-}\left(E^{k+1}+E_{I_{j}} ; F_{c_{j}}\right)\right]} \\
& +\frac{p_{-+}\left(E^{k+1}+E_{I_{j}} ; F_{c_{j}}\right) \Delta t}{1+\Delta t\left[p_{-+}\left(E^{k+1}+E_{I_{j}} ; F_{c_{j}}\right)+p_{+-}\left(E^{k+1}+E_{I_{j}} ; F_{c_{j}}\right)\right]}
\end{aligned}
$$




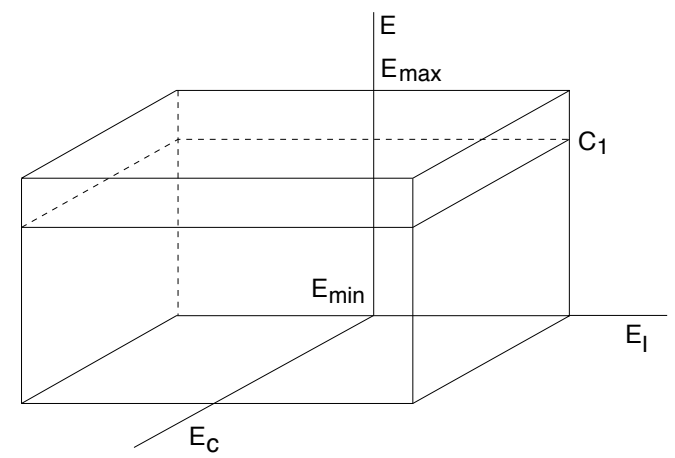

Figure 3: Structure of the 3-D array $\mathcal{C}_{1}$ and 2-D array $C_{1}$ for specified field value.

Remark 1. Because the constants $c_{1}$ and $c_{2}$ in (25) depend on field values but not explicitly on time, they can be evaluated offline and stored for highly efficient subsequent model implementation. To achieve this, one can construct 3 -dimensional arrays $\mathcal{C}_{1}$ and $\mathcal{C}_{2}$ whose coordinates are $F_{c}, E_{I}$ and $E$ with values at the quadrature points $\left(F_{c_{i}}, E_{I_{j}}\right)$ and field values $E^{\ell}$ uniformly distributed between minimum and maximum field values $E_{\min }, E_{\max }$ of operation; see Figure 3 . During model execution - e.g., during parameter estimation or model-based control implementation — one would access the 2-dimensional, $N_{i} \times N_{j}$, arrays $C_{1}^{\ell}=\mathcal{C}_{1}\left(:,:, E^{\ell}\right)$ and $C_{2}^{\ell}=\mathcal{C}_{2}\left(:,:, E^{\ell}\right)$, for $E^{\ell}$ nearest the specified field value $E^{k+1}$, and update $X_{+}$using the componentwise matrix multiplication and summation

$$
X_{+}^{k+1}=C_{1}^{\ell} \cdot \times X^{k}+C_{2}^{\ell} .
$$

When combined with (26), the efficiency of implementing the thermal relaxation model is the same as that of Algorithm 2.6.4 on page 117 of [18] for the negligible relaxation model.

An alternative is to form arrays $\mathcal{P}_{-+}, \mathcal{P}_{+-}$storing the likelihoods at the $N_{i} \times N_{j}$ quadrature points and predefined field values. The matrices $C_{1}^{\ell}, C_{2}^{\ell}$ can then be constructed using the operations defined in (28). This is slightly less efficient but can preferable if memory is limited and $\mathcal{P}_{-+}, \mathcal{P}_{+-}$are used for the Jacobian construction described in Section 4 . It is also advantageous if variable stepsizes $\Delta t$ are required for control implementation.

\subsubsection{Polarization-Strain Model}

From (8)-(10), it follows that the discretized polarization and strain relations can be expressed as

$$
\begin{aligned}
& P(E, \sigma)=\chi^{\sigma} E+\sum_{\alpha= \pm, 90}\left(\sigma d_{\alpha}+P_{R}^{\alpha}\right) V^{T} X_{\alpha}(t ; E, \sigma) W \\
& \varepsilon(E, \sigma)=s^{E} \sigma+\sum_{\alpha= \pm, 90}\left(E d_{\alpha}+\varepsilon_{R}^{\alpha}\right) V^{T} X_{\alpha}(t ; E, \sigma) W
\end{aligned}
$$

where $V$ and $W$ are defined in (27). The $N_{i} \times N_{j}$ matrices $X_{\alpha}(E, \sigma)$ are defined componentwise by

$$
\left[X_{\alpha}(t ; E, \sigma)\right]_{i j}=x_{\alpha}\left(t ; E+E_{I_{j}}, \sigma, F_{c_{i}}\right), \alpha= \pm, 90
$$

where $x_{ \pm, 90}$ are solutions of (11).

For discrete time, field and stress values $t_{k}=k \Delta t, E^{k}=E\left(t_{k}\right)$ and $\sigma^{k}=\sigma\left(t_{k}\right)$, we let $x_{\alpha}^{k}=$ $x_{\alpha}\left(t_{k} ; E^{k}+E_{I}, \sigma^{k}, F_{c}\right)$ and $\left[X_{\alpha}^{k}\right]_{i j}=x_{\alpha}\left(t_{k} ; E^{k}+E_{I_{j}}, \sigma^{k}, F_{c_{i}}\right)$ denote the associated dipole fractions 
and matrix of dipole fractions evaluated at quadrature points. An implicit Euler discretization then yields

$$
\begin{aligned}
& a_{11}^{k+1} x_{-}^{k+1}+a_{12}^{k+1} x_{+}^{k+1}=x_{-}^{k}+a_{13}^{k+1} \\
& a_{21}^{k+1} x_{-}^{k+1}+a_{22}^{k+1} x_{+}^{k+1}=x_{+}^{k}+a_{23}^{k+1}
\end{aligned}
$$

where

$$
\begin{aligned}
& a_{11}^{k+1}=1+\Delta t\left(p_{-90}+p_{-+}+p_{90-}\right) \quad, \quad a_{21}^{k+1}=-\Delta t\left(p_{-+}+p_{90+}\right) \\
& a_{12}^{k+1}=-\Delta t\left(p_{+-}+p_{90-}\right) \quad, a_{22}^{k+1}=1+\Delta t\left(p_{+90}+p_{+-}+p_{90+}\right) \\
& a_{13}^{k+1}=\Delta t p_{90-} \quad, \quad a_{23}^{k+1}=\Delta t p_{90+} .
\end{aligned}
$$

We note that like the dipole fractions, these constants depend on $\left(E, E_{I}, F_{c}\right)$; e.g., $a_{13}^{k+1}\left(E, E_{I}, F_{c}\right)=$ $\Delta t p_{90-}\left(E^{k+1}+E_{I} ; F_{c}\right)$.

Using Cramer's rule, one can solve (31) to obtain

$$
\begin{aligned}
& x_{-}^{k+1}=c_{1}^{k+1} x_{-}^{k}+c_{2}^{k+1} x_{+}^{k}+c_{3}^{k+1} \\
& x_{+}^{k+1}=d_{1}^{k+1} x_{-}^{k}+d_{2}^{k+1} x_{+}^{k}+d_{3}^{k+1}
\end{aligned}
$$

where

$$
\begin{aligned}
& c_{1}^{k+1}=\frac{a_{22}^{k+1}}{\operatorname{det}} \quad, \quad c_{2}^{k+1}=\frac{-a_{12}^{k+1}}{\operatorname{det}} \quad, \quad c_{3}^{k+1}=\frac{1}{\operatorname{det}}\left[a_{22}^{k+1} a_{13}^{k+1}-a_{12}^{k+1} a_{23}^{k+1}\right] \\
& d_{1}^{k+1}=\frac{-a_{21}^{k+1}}{\operatorname{det}} \quad, \quad d_{2}^{k+1}=\frac{a_{11}^{k+1}}{\operatorname{det}} \quad, \quad d_{3}^{k+1}=\frac{1}{\operatorname{det}}\left[a_{11}^{k+1} a_{23}^{k+1}-a_{13}^{k+1} a_{21}^{k+1}\right] \\
& \operatorname{det}=a_{11}^{k+1} a_{22}^{k+1}-a_{12}^{k+1} a_{21}^{k+1} .
\end{aligned}
$$

To facilitate efficient implementation, we follow the strategy detailed in Remark 1 and construct 3-D arrays $\mathcal{C}_{1}-\mathcal{D}_{3}$ comprised of the constants $c_{1}-d_{3}$ evaluated at the quadrature points $\left(F_{c_{i}}, E_{I_{j}}\right)$ and field values $E^{\ell}$ uniformly distributed between the minimum and maximum field values $E_{\text {min }}$ and $E_{\max }$; e.g., $\left[\mathcal{C}_{2}\right]_{i j}=\Delta t\left[p_{+-}\left(E^{\ell}+E_{I_{j}} ; F_{c_{i}}\right)+p_{90-}\left(E^{\ell}+E_{I_{j}} ; F_{c_{i}}\right)\right] / \operatorname{det}\left(E^{\ell}, E_{I_{j}}, F_{c_{i}}\right)$. During model implementation, one then accesses the $N_{i} \times N_{j}$ matrices $C_{1}^{\ell}, C_{2}^{\ell}, C_{3}^{\ell}, D_{1}^{\ell}, D_{2}^{\ell}, D_{3}^{\ell}$, nearest to the specified field value $E^{k}$. The matrices of dipole fractions are then updated using the relation

$$
\begin{aligned}
& X_{+}^{k+1}=C_{1}^{\ell} \cdot \times X_{+}^{k}+C_{2}^{\ell} \cdot \times X_{-}^{k}+C_{3}^{\ell} \\
& X_{-}^{k+1}=D_{1}^{\ell} \cdot \times X_{+}^{k}+D_{2}^{\ell} \cdot \times X_{-}^{k}+D_{3}^{\ell} \\
& X_{90}^{k+1}=I-X_{+}^{k+1}-X_{-}^{k+1}
\end{aligned}
$$

which again involves only componentwise matrix multiplication and summation.

\section{Parameter Estimation Problem}

The data for the parameter estimation problem is taken to be either polarization measurements $P_{i}$ or polarization-strain pairs $\left\{P_{i}, \varepsilon_{i}\right\}$ corresponding to $N_{d}$ field values $E\left(t_{i}\right)$. The model response with parameters $p$ for the same field values and a constant prestress $\sigma_{0}$ is denoted by $P\left(E\left(t_{i}\right), \sigma_{0} ; p\right)$ and $\varepsilon\left(E\left(t_{i}\right), \sigma_{0} ; p\right)$.

To calibrate the $180^{\circ}$ polarization model, summarized in Table 2, with the parameter $p$ given by (15), we minimize the functional

$$
J(p)=\frac{1}{2} \sum_{i=1}^{N_{d}} e^{2}\left(t_{i} ; p\right)
$$


where

$$
e\left(t_{i} ; p\right)=P\left(E\left(t_{i}\right), \sigma_{0} ; p\right)-P_{i} .
$$

We first note that the magnitudes of the first four parameters $\left\{\eta, P_{R}, \gamma, \tau\right\}$ can vary from $10^{-4}$ to $10^{8}$ so the performance of optimization algorithms is significantly improved by scaling the parameters by dividing by their magnitudes. To accomplish this, we form a vector $s$, whose components are the scale of each parameter, and minimize instead

$$
J(q)=\frac{1}{2} \sum_{i=1}^{N_{d}} e^{2}\left(t_{i} ; q . \times s\right)
$$

where $q=p . / s$ and.$/$ and.$\times$ respectively denote componentwise division and multiplication. Because the components of $q$ are on the order of unity, (34) is more readily mimimized using standard optimization software.

To estimate parameters for the combined polarization and strain model (8), we employ the functional

$$
\begin{aligned}
J(q) & =\frac{1}{2} \sum_{i=1}^{N_{d}} w_{P} e_{P}^{2}\left(t_{i} ; q \cdot \times s\right)+\frac{1}{2} \sum_{i=1}^{N_{d}} w_{\varepsilon} e_{\varepsilon}^{2}\left(t_{i} ; q \cdot \times s\right) \\
& =\frac{1}{2} \sum_{i=1}^{2 N_{d}} R_{i}(q)
\end{aligned}
$$

where

$$
\begin{aligned}
& e_{P}\left(t_{i} ; q . \times s\right)=P\left(E\left(t_{i}\right), \sigma_{0} ; q . \times s\right)-P_{i} \\
& e_{\varepsilon}\left(t_{i} ; q . \times s\right)=\varepsilon\left(E\left(t_{i}\right), \sigma_{0} ; q \cdot \times s\right)-\varepsilon_{i} \\
& R_{i}(q)= \begin{cases}w_{P} e_{P}^{2}\left(t_{i} ; q . \times s\right), & i=1, \cdots, N_{d} \\
w_{\varepsilon} e_{\varepsilon}^{2}\left(t_{i} ; q \times s\right), & i=N_{d}+1, \cdots, 2 N_{d} .\end{cases}
\end{aligned}
$$

Here $p=q . \times s$ is given by (7). Since polarization is typically on the order of $10^{-1}$ and strain on the order of $10^{-3}$, the weights $w_{P}$ and $w_{\varepsilon}$ are chosen to balance the polarization and strain components of (37).

The behavior of the functionals $J(q)$ is typical of those arising in parameter estimation problems; they have shallow slopes near the global minimum (if it exists) and they are typically nonconvex with multiple local minima.

One possibility is to employ stochastic optimization techniques such as genetic algorithms, simulated annealing, and differential evolution [22]. These techniques reduce the reliance on accurate initial parameter estimates and, in theory, provide global convergence. However, their convergence rates are slower - they may require infinite time for convergence - and, because they are nondeterministic, multiple optimizations can yield varying final parameter values.

Alternatively, one can employ gradient-based techniques such as the interior-reflective Newton, Levenberg-Marquardt, or sequential quadratic programming methods employed in the MATLAB routines lsqnonlin and fmincon. The efficiency and success of gradient-based optimization methods is predicated on determining good initial parameter estimates and being able to accurately determine gradients. We show next that analytic gradient expressions can be constructed for the homogenized energy model and, in Section 4.2, we use physical aspects of the model to develop data-driven techniques to provide initial parameter estimates based on measured properties of polarization and strain data. In combination, this permits routines, such as lsqnonlin which is used to optimize (32) and (33), to be implemented in a matter of minutes. 


\subsection{Analytic Jacobian or Gradient Relations}

Most commercial optimization packages provide the option for constructing the Jacobian using finite difference approximation or providing an analytic expression. The former option is certainly feasible for optimizing (34) or (35) but it will be significantly slower and slightly less accurate than optimization using the analytic Jacobian. We illustrate first the computation of the Jacobian for the polarization model (16) with the functional (34) since it is simpler than that for the combined polarization-strain model.

\section{Polarization Model}

The Jacobian corresponding to (34) is

$$
\mathcal{J}=\left[\begin{array}{cccc}
\frac{\partial e\left(t_{1} ; p\right)}{\partial \eta} & \frac{\partial e\left(t_{1} ; p\right)}{\partial P_{R}} & \cdots & \frac{\partial e\left(t_{1} ; p\right)}{\partial \beta_{k}} \\
\vdots & \vdots & & \vdots \\
\frac{\partial e\left(t_{N_{d}} ; p\right)}{\partial \eta} & \frac{\partial e\left(t_{N_{d}} ; p\right)}{\partial P_{R}} & \cdots & \frac{\partial e\left(t_{N_{d}} ; p\right)}{\partial \beta_{k}}
\end{array}\right]
$$

where $e\left(t_{i} ; p\right)$ is defined in $(33)$. We note that

$$
[\mathcal{J}]_{i j}=\frac{\partial e\left(t_{i} ; p\right)}{\partial q_{j}}=\frac{\partial}{\partial q_{j}} P\left(E\left(t_{i}\right), \sigma_{0} ; p\right)=\frac{\partial}{\partial p_{j}} P\left(E\left(t_{i}\right), \sigma_{0} ; p\right) \frac{d p_{j}}{d q_{j}}=\frac{\partial}{\partial p_{j}} P\left(E\left(t_{i}\right), \sigma_{0} ; p\right) s_{j}
$$

so the Jacobian is simply the gradient or sensitivity of $P$ with respect to the parameters. For the discretized model, these derivatives have the form

$$
\begin{aligned}
& \frac{\partial P}{\partial \eta}=-\frac{E}{\eta^{2}} \quad, \frac{\partial P}{\partial P_{R}}=-1+2 V^{T}\left[X_{+}\right] W+2 P_{R} V^{T}\left[\partial_{P_{R}} X_{+}\right] W \\
& \frac{\partial P}{\partial \gamma}=2 P_{R} V^{T}\left[\partial_{\gamma} X_{+}\right] W \quad, \frac{\partial P}{\partial \tau}=2 P_{R} V^{T}\left[\partial_{\tau} X_{+}\right] W \\
& \frac{\partial P}{\partial \alpha_{k}}=\frac{2 P_{R}}{\sum_{\ell} \alpha_{\ell}}\left(\mathcal{V}_{k}-V\right)^{T}\left[X_{+}\right] W \quad, \quad \frac{\partial P}{\partial \beta_{k}}=\frac{2 P_{R}}{\sum_{\ell} \beta_{\ell}} V^{T}\left[X_{+}\right]\left(\mathcal{W}_{k}-W\right)
\end{aligned}
$$

where $\left[V^{T}\right]_{i}=v_{i} \nu_{c}\left(F_{c_{i}}\right),[W]_{j}=w_{j} \nu_{I}\left(E_{I_{j}}\right)$, as indicated in $(27)$, and $\left[\mathcal{V}_{k}^{T}\right]_{i}=v_{i} \phi_{k}\left(F_{c_{i}}\right),\left[\mathcal{W}_{k}\right]_{j}=$ $w_{j} \varphi_{k}\left(E_{I_{j}}\right)$. The components of the $N_{i} \times N_{j}$ matrices $\left[\partial_{\xi} X_{+}\right]_{i j}=\frac{\partial x_{+}}{\partial \xi}\left(t ; E(t)+E_{I_{j}}, \sigma_{0} ; F_{c_{i}}\right)$ for $\xi=P_{R}, \gamma, \tau$, can be found by differentiating the evolution equation (19) and switching the order of differentiation to obtain the differential equations

$$
\begin{aligned}
& \dot{x}^{P_{R}}=-\left(\partial_{P_{R}} p_{+-}+\partial_{P_{R}} p_{-+}\right) x_{+}-\left(p_{+-}+p_{-+}\right) x^{P_{R}}+\partial_{P_{R}} p_{-+} \\
& \dot{x}^{\gamma}=-\left(\partial_{\gamma} p_{+-}+\partial_{\gamma} p_{-+}\right) x_{+}-\left(p_{+-}+p_{-+}\right) x^{\gamma}+\partial_{\gamma} p_{-+} \\
& \dot{x}^{\tau}=-\left(\partial_{\tau} p_{+-}+\partial_{\tau} p_{-+}\right) x_{+}-\left(p_{+-}+p_{-+}\right) x^{\tau}+\partial_{\tau} p_{-+}
\end{aligned}
$$

in the variables $x^{P_{R}} \equiv \frac{\partial x_{+}}{\partial P_{R}}, x^{\gamma} \equiv \frac{\partial x_{+}}{\partial \gamma}$ and $x^{\tau} \equiv \frac{\partial x_{+}}{\partial \tau}$ with

$$
\begin{aligned}
& \partial_{P_{R}} p_{+-}=\left\{\begin{array}{ccc}
-\gamma \frac{\left(E+E_{c}\right)^{2}}{2 E_{c}} p_{+-} & , \quad E \leq E_{c} \\
0 & , \quad E>E_{c}
\end{array}, \quad \partial_{P_{R}} p_{-+}=\left\{\begin{array}{cl}
-\gamma \frac{\left(E-E_{c}\right)^{2}}{2 E_{c}} p_{-+} & , \quad E \leq E_{c} \\
0 & , E>E_{c}
\end{array}\right.\right. \\
& \partial_{\gamma} p_{+-}=-\Delta G_{+-}^{a} p_{+-} \quad, \partial_{\gamma} p_{-+}=-\Delta G_{-+}^{a} p_{-+} \\
& \partial_{\tau} p_{+-}=-\frac{1}{\tau} p_{+-} \quad, \quad \partial_{\tau} p_{-+}=-\frac{1}{\tau} p_{-+} .
\end{aligned}
$$

Given values of $x_{+}$and $x_{-}$, the differential equations (36) can be solved using an implicit Euler method analogous to that described in Section 3.2.1 to approximate the solution of (19). 
Remark 2. The Jacobian construction can be facilitated by the use of lookup tables, in a manner analogous to that described in Section 3.2.1, to avoid the repeated evaluation of the likelihoods $p_{+-}$and $p_{-+}$for all of the quadrature points $\left(F_{c_{i}}, E_{I_{j}}\right)$. Alternatively, if memory is limited, one can construct two arrays $\mathcal{P}_{-+}, \mathcal{P}_{+-}$storing the likelihoods at field values and quadrature points in a manner similar to that discussed in Remark 1 of Section 3. If memory is plentiful, it will be more efficient to construct lookup tables associated with the quantities defined in (38).

\section{Polarization-Strain Model}

The construction of the Jacobian for the functional (33) is analogous but slightly more complicated since it involves more parameters $p=\left[P_{R}^{+}, \varepsilon_{R}^{+}, \varepsilon_{R}^{90}, \chi^{\sigma}, d_{+}, s^{E}, \gamma, \tau_{90}, \tau_{180}, \alpha_{k}, \beta_{k}\right]$. We first note that the model (29) exhibits the parameter dependencies

$$
\begin{aligned}
& P(E, \sigma)=\chi^{\sigma} E+\sum_{\alpha= \pm, 90}\left(\sigma d_{\alpha}+P_{R}^{\alpha}\right) V^{T}\left(\alpha_{k}\right) X_{\alpha}(t ; E, \sigma, \hat{p}) W\left(\beta_{k}\right) \\
& \varepsilon(E, \sigma)=s^{E} \sigma+\sum_{\alpha= \pm, 90}\left(E d_{\alpha}+\varepsilon_{R}^{\alpha}\right) V^{T}\left(\alpha_{k}\right) X_{\alpha}(t ; E, \sigma, \hat{p}) W\left(\beta_{k}\right)
\end{aligned}
$$

where $V$ and $W$ are defined in (27), $X_{\alpha}$ is defined in (30), and

$$
\hat{p}=\left[P_{R}^{+}, \varepsilon_{R}^{+}, \varepsilon_{R}^{90}, d_{+}, \gamma, \tau_{90}, \tau_{180}\right] .
$$

With the assumptions(4) and definitions $\left[\mathcal{V}_{k}^{T}\right]_{i}=v_{i} \phi_{k}\left(F_{c_{i}}\right),\left[\mathcal{W}_{k}\right]_{j}=w_{j} \varphi_{k}\left(E_{I_{j}}\right)$, it follows that

$$
\begin{aligned}
& \frac{\partial P}{\partial P_{R}^{+}}=V^{T}\left[X_{+}\right] W-V^{T}\left[X_{-}\right] W+\sum_{\alpha= \pm, 90}\left(\sigma d_{\alpha}+P_{R}^{\alpha}\right) V^{T}\left[\partial_{P_{R}^{+}} X_{\alpha}\right] W \\
& \frac{\partial P}{\partial d_{+}}=\sigma V^{T}\left[X_{+}\right] W-\sigma V^{T}\left[X_{-}\right] W+\sum_{\alpha= \pm, 90}\left(\sigma d_{\alpha}+P_{R}^{\alpha}\right) V^{T}\left[\partial_{d_{+}} X_{\alpha}\right] W \\
& \frac{\partial P}{\partial \zeta}=\sum_{\alpha= \pm, 90}\left(\sigma d_{\alpha}+P_{R}^{\alpha}\right) V^{T}\left[\partial_{\zeta} X_{\alpha}\right] W \\
& \frac{\partial P}{\partial \alpha_{k}}=\sum_{\alpha= \pm, 90} \frac{\left(\sigma d_{\alpha}+P_{R}^{\alpha}\right)}{\sum_{\ell} \alpha_{\ell}}(\mathcal{V}-V)^{T}\left[X_{\alpha}\right] W \quad, \quad \frac{\partial P}{\partial \beta_{k}}=\sum_{\alpha= \pm, 90} \frac{\left(\sigma d_{\alpha}+P_{R}^{\alpha}\right)}{\sum_{\ell} \beta_{\ell}} V^{T}\left[X_{\alpha}\right](\mathcal{W}-W) \\
& \frac{\partial P}{\partial \chi^{\sigma}}=E \quad, \quad \frac{\partial P}{\partial s^{E}}=0
\end{aligned}
$$

for $\zeta=\varepsilon_{R}^{+}, \varepsilon_{R}^{90}, \gamma, \tau_{90}$ and $\tau_{180}$. Differentiation of the strain relations yields similar terms.

We next discuss the construction of the $N_{i} \times N_{j}$ matrices $\left[\partial_{\xi} X_{\alpha}\right]_{i j}=\frac{\partial x_{\alpha}}{\partial \xi}\left(t ; E(t)+E_{I_{j}}, \sigma_{0} ; F_{c_{i}}\right)$ where $\xi=P_{R}^{+}, d_{+}, \varepsilon_{R}^{+}, \varepsilon_{R}^{90}, \gamma, \tau_{90}$ and $\tau_{180}$. Differentiation of (11) with respect to $\xi$ and switching the order of differentiation yields the fourteen differential equations

$$
\begin{aligned}
\dot{x}_{-}^{\xi}= & -\frac{\partial}{\partial \xi}\left(p_{-90}+p_{-+}+p_{90-}\right) x_{-}-\left(p_{-90}+p_{-+}+p_{90-}\right) x_{-}^{\xi} \\
& +\frac{\partial}{\partial \xi}\left(p_{+-}-p_{90-}\right) x_{+}+\left(p_{+-}-p_{90-}\right) x_{+}^{\xi}+\frac{\partial p_{90-}}{\partial \xi} \\
\dot{x}_{+}^{\xi}= & \frac{\partial}{\partial \xi}\left(p_{-+}-p_{90+}\right) x_{-}+\left(p_{-+}-p_{90+}\right) x_{-}^{\xi} \\
& -\frac{\partial}{\partial \xi}\left(p_{+90}+p_{+-}+p_{90+}\right) x_{-}-\left(p_{+90}+p_{+-}+p_{90+}\right) x_{-}^{\xi}+\frac{\partial p_{90+}}{\partial \xi}
\end{aligned}
$$


in the variables $x_{+}^{\xi} \equiv \frac{\partial x_{+}}{\partial \xi}, x_{-}^{\xi} \equiv \frac{\partial x_{-}}{\partial \xi}$. For an input field value $E\left(t_{k}\right), x_{+}$and $x_{-}$can be determined using the techniques detailed in Section 3.2.2, and the solution to (39) can be approximated using an implicit Euler discretization and Cramer's rule in a manner analogous to that detailed in Section 3.2.2.

To evaluate the derivatives of the likelihoods

$$
p_{\alpha \beta}=\frac{1}{\tau_{\alpha \beta}} e^{-\gamma \Delta G_{\alpha \beta}^{a}}=\left\{\begin{array}{cc}
\frac{1}{\tau_{\alpha \beta}} e^{-\gamma \Delta G_{0}\left(1-F_{\alpha \beta} / F_{c}\right)^{2}} & , \quad F_{\alpha \beta} \leq F_{c} \\
0 & , \quad F_{\alpha \beta}>F_{c}
\end{array}\right.
$$

we note that

$$
\frac{\partial p_{\alpha \beta}}{\partial \gamma}=-\Delta G_{\alpha \beta}^{a} p_{\alpha \beta} \quad, \quad \frac{\partial p_{\alpha \beta}}{\partial \tau_{\alpha \beta}}=\frac{-1}{\tau_{\alpha \beta}} p_{\alpha \beta}
$$

and

$$
\frac{\partial p_{\alpha \beta}}{\partial \xi}=\left\{\begin{array}{cl}
\frac{2 \gamma \Delta G_{0}}{F_{c}}\left(1-F_{\alpha \beta} / F_{c}\right) p_{\alpha \beta} \frac{\partial F_{\alpha \beta}}{\partial \xi} & , \quad F_{\alpha \beta} \leq F_{c} \\
0 & , \quad F_{\alpha \beta}>F_{c} .
\end{array}\right.
$$

The values of $\frac{\partial F_{\alpha \beta}}{\partial \xi}$ resulting from the relations (13) are compiled in Table 3.

\begin{tabular}{|c|c|c|c|c|c|c|}
\hline$\xi$ & $90+$ & +90 & $90-$ & -90 & +- & -+ \\
\hline$P_{R}^{+}$ & $E$ & $-E$ & $-E$ & $E$ & $-2 E$ & $2 E$ \\
\hline$\varepsilon_{R}^{+}$ & $\sigma$ & $-\sigma$ & $\sigma$ & $-\sigma$ & 0 & 0 \\
\hline$\varepsilon_{R}^{90}$ & $-\sigma$ & $\sigma$ & $-\sigma$ & $\sigma$ & 0 & 0 \\
\hline$d_{+}$ & $E \sigma$ & $-E \sigma$ & $-E \sigma$ & $E \sigma$ & $-2 E \sigma$ & $2 E \sigma$ \\
\hline
\end{tabular}

Table 3: Values of the derivatives $\frac{\partial F_{\alpha \beta}}{\partial \xi}$.

\subsection{Data-Driven Techniques for Initial Parameter Estimates}

An ideal data set for obtaining data-driven initial parameter estimates and optimization-based final parameter values has two components: (i) saturated major loop and biased minor loop polarization and strain data, and (ii) creep polarization or strain data at two or more field values as depicted in Figure 4(a) and (b). If creep data is not available, frequency-dependent data, such as that depicted in Figure 4(c) can be used to provide an initial estimate for $\tau_{90}$.

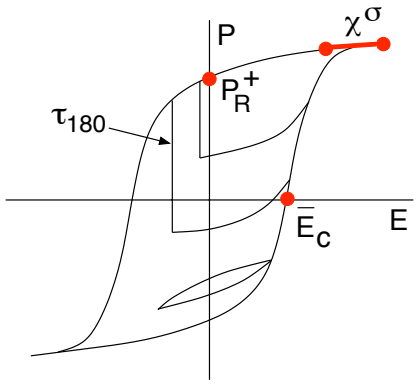

(a)

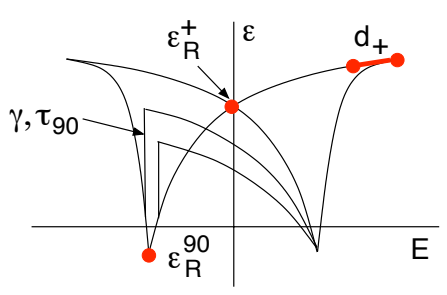

(b)

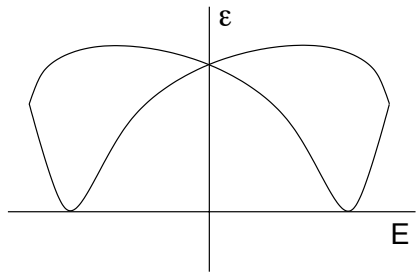

(c)

Figure 4: (a) and (b) Saturated major loop, minor loop, and creep data used to determine initial estimates for $P_{R}^{+}, \varepsilon_{R}^{+}, \varepsilon_{R}^{90}, \chi^{\sigma}, d_{+}, \gamma, \tau_{90}$ and $\tau_{180}$. (c) Frequency-dependent strain data used to determine initial estimate for $\tau_{90}$ or $\tau_{180}$. 

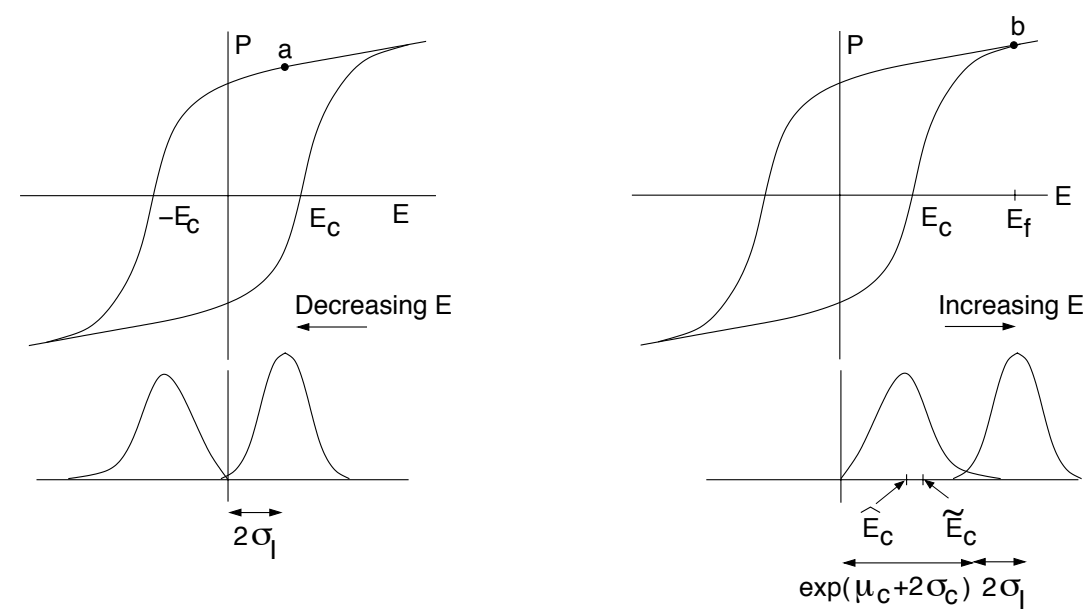

Figure 5: Polarization and field values used to obtain initial estimates for $\bar{F}_{c}, \sigma_{c}, \sigma_{I}$.

As noted in (7), the parameters to be estimated are $p=\left[P_{R}^{+}, \varepsilon_{R}^{+}, \varepsilon_{R}^{90}, \chi^{\sigma}, d_{+}, s^{E}, \gamma, \tau_{90}, \tau_{180}, \alpha_{k}, \beta_{k}\right]$. The representations (5) for the densities $\nu_{c}\left(F_{c}\right)$ and $\nu_{I}\left(E_{I}\right)$ additionally requires estimation of the coefficients $\bar{p}=\left[\bar{F}_{c}, \sigma_{c}, \sigma_{I}\right]$.

Data-driven techniques to estimate initial values for $P_{R}^{+}, \varepsilon_{R}^{+}, \varepsilon_{R}^{90}, \chi^{\sigma}, d_{+}, \bar{E}_{c}, \bar{F}_{c}, \sigma_{c}, \sigma_{I}$ are summarized in Table 4 with reference to Figures 4 and 5 . The values of $\sigma_{I}$ and $\sigma_{c}$ are then used to construct the vectors $\vec{\sigma}_{I}=\left\{2^{k} \sigma_{c}\right\}$ and $\vec{\sigma}_{c}=\left\{2^{k} \sigma_{c}\right\}$ for the density basis functions $\phi_{k}\left(E_{I}\right)$ and $\varphi_{k}\left(F_{c}\right)$. One strategy to obtain initial values for the coefficients $\alpha_{k}$ and $\beta_{k}$ is simply to take $\alpha_{k}=\beta_{k}=1$. Documented values provide an initial estimate for $s^{E}$.

\section{Initial Estimates for $\gamma, \tau_{90}$ and $\tau_{180}$ Based on Creep Data}

Creep data collected at two or more fixed fields, as indicated by the thick lines in Figure 6, can be used to determine initial estimates for $\gamma, \tau_{90}$ and $\tau_{180}$. Whereas either strain or polarization data can be employed, the former exhibits more sensitivity to $90^{\circ}$ switching so we employ it here. The fixed field values are denoted by $\bar{E}_{1}$ and $\bar{E}_{2}$ as indicated by (1) and (2) for the strains plotted in Figure 6 . We note that $\bar{E}_{1}$ designated by (1) satisfies $\bar{E}_{1}<-E_{c}$. Finally, let $t_{0}$ designate the time at which the field was fixed, let $\varepsilon_{1}, \varepsilon_{2}$ and $\varepsilon_{3}$ designate measured strain values at equally spaced subsequent times $t_{1}=t_{0}+\Delta t, t_{2}=t_{0}+2 \Delta t$ and $t_{3}=t_{0}+3 \Delta t$, and let $e_{2}=\varepsilon_{2}-\varepsilon_{1}, e_{3}=\varepsilon_{3}-\varepsilon_{2}$.

Assumption 1. We assume that the decay behavior of dipole fractions can be adequately approximated by neglecting interaction fields and assuming that switches occur at $\pm E_{c}$. For a fixed field $E>E_{c}$, we assume that $x_{-}=0$ and that $p_{90_{-}}=p_{+90}=p_{+-}=0$. Similarly, for $E<-E_{c}$, we assume that $x_{+}=0$ and that $p_{-90}=p_{90+}=p_{-+}=0$.

With these simplifying assumptions, it follows from (2) that

$$
\begin{aligned}
& \dot{x}_{-}=-p_{90-} x_{-}+p_{90-} \quad, \quad E<-E_{c} \\
& \dot{x}_{+}=-p_{90+} x_{+}+p_{90+} \quad, \quad E>E_{c}
\end{aligned}
$$

For fixed fields, (40) has the analytic solution

$$
\begin{array}{ll}
x_{-}(t, E)=1-\left(x_{-}\left(t_{0}, E\right)-1\right) e^{-\left(t-t_{0}\right) p_{90-}(E)}, & E<-E_{c} \\
x_{+}(t, E)=1-\left(x_{+}\left(t_{0}, E\right)-1\right) e^{-\left(t-t_{0}\right) p_{90+}(E)} & , \quad E>E_{c} .
\end{array}
$$




\begin{tabular}{|c|c|c|}
\hline Parameter & Data or Source & Justification \\
\hline$P_{R}^{+}$ & Remanence polarization & \\
\hline$\varepsilon_{R}^{+}$ & Remanence strain & \\
\hline$\varepsilon_{R}^{90}$ & Minimum strain & \\
\hline$\chi^{\sigma}$ & $\begin{array}{l}\text { Slope of } E-P \text { curve prior to switch- } \\
\text { ing. }\end{array}$ & $\begin{array}{l}\text { From the relation } P=\chi^{\sigma} E=\epsilon_{0}\left(\epsilon_{r}-1\right) E \text {, } \\
\text { it follows that the relative permittivity } \epsilon_{r} \\
\text { and } \chi^{\sigma} \text { satisfy } \chi^{\sigma}=\epsilon_{0}\left(\epsilon_{r}-1\right) \text { where } \epsilon_{0}= \\
8.85 \times 10^{-12} \mathrm{~F} / \mathrm{m} \text { is the permittivity of free } \\
\text { space. }\end{array}$ \\
\hline$d_{+}$ & Slope of $E$ - $\varepsilon$ curve prior to switching. & $\begin{array}{l}\text { Can be compared to documented material } \\
\text { values. }\end{array}$ \\
\hline$s^{E}$ & Slope of $\sigma-\varepsilon$ curve. & $\begin{array}{l}\text { Can be compared to documented material } \\
\text { values. }\end{array}$ \\
\hline$\gamma, \tau_{90}, \tau_{180}$ & Creep data as shown in Figure 4. & $\begin{array}{l}\left.\gamma \text { and } \tau_{90} \text { given by solution of ( } 43\right) \text { using data } \\
\text { from two fixed fields } \bar{E}_{1} \text { and } \bar{E}_{2} \text {. We typi- } \\
\text { cally take } \tau_{180}=\frac{1}{10} \tau_{90} \text {. }\end{array}$ \\
\hline$\alpha_{k}=\beta_{k}=1$ & A priori choice & \\
\hline $\begin{array}{l}\bar{E}_{c} \\
\bar{F}_{c} \\
2 P_{R}^{+} \bar{E}_{c}\end{array}$ & Coercive field $E_{c}$ & $\begin{array}{l}\text { Whereas the mode } \hat{E}_{c} \text { most closely coincides } \\
\text { with } E_{c} \text { (see Appendix B), by approximating } \\
\text { with the median } \tilde{E}_{c} \text {, the relation } \tilde{E}_{c}=e^{\mu_{c}} \\
\text { from }(50) \text { can be used to obtain } \mu_{c}=\bar{E}_{c} .\end{array}$ \\
\hline$\sigma_{I}$ & $a$ & $\begin{array}{l}\text { Switching begins when } \nu_{I}\left(E_{I}\right) \text { intersects } \\
\nu_{c}\left(-E_{c}\right) \text { with } 2 \sigma_{I} \text { specifying the } 94.5 \% \text { con- } \\
\text { fidence level. }\end{array}$ \\
\hline$\sigma_{c}$ & $\begin{array}{l}E_{f}: \text { Polarization where switching } \\
\text { completes for increasing } E \text {. }\end{array}$ & $\begin{array}{l}\text { Occurs when } \nu_{I}\left(E_{I}\right) \text { quits intersecting } \\
\nu_{c}\left(E_{c}\right) \text { at } 94.5 \% \text { confidence level. From }(51) \text {, } \\
\text { this yields } \sigma_{c}=\frac{1}{2} \ln \left(\left(E_{f}-2 \sigma_{I}\right) / \tilde{E}_{c}\right) \text { since } \\
e^{\mu_{c}+2 \sigma_{c}}+2 \sigma_{I}=E_{f} .\end{array}$ \\
\hline
\end{tabular}

Table 4: Data-driven algorithms for initial parameter estimates with reference to Figures 4-6.

From Assumption 1, it follows that

$$
\varepsilon(E, \sigma) \approx s^{E} \sigma+E \sum_{\alpha= \pm, 90} d_{\alpha} x_{\alpha}+\sum_{\alpha= \pm, 90} \varepsilon_{R}^{\alpha} x_{\alpha}
$$

which, when combined with the physical assumptions in (4), yields

$$
\begin{aligned}
\frac{e_{3}}{e_{2}} & =\frac{\left[\left(E d_{+}+\varepsilon_{R}^{+}-\varepsilon_{R}^{90}\right) x_{+}\left(t_{3}, E\right)+\varepsilon_{R}^{90}\right]-\left[\left(E d_{+}+\varepsilon_{R}^{+}-\varepsilon_{R}^{90}\right) x_{+}\left(t_{2}, E\right)+\varepsilon_{R}^{90}\right]}{\left[\left(E d_{+}+\varepsilon_{R}^{+}-\varepsilon_{R}^{90}\right) x_{+}\left(t_{2}, E\right)+\varepsilon_{R}^{90}\right]-\left[\left(E d_{+}+\varepsilon_{R}^{+}-\varepsilon_{R}^{90}\right) x_{+}\left(t_{1}, E\right)+\varepsilon_{R}^{90}\right]} \\
& =\frac{x_{+}\left(t_{3}, E\right)-x_{+}\left(t_{2}, E\right)}{x_{+}\left(t_{2}, E\right)-x_{+}\left(t_{1}, E\right)}
\end{aligned}
$$




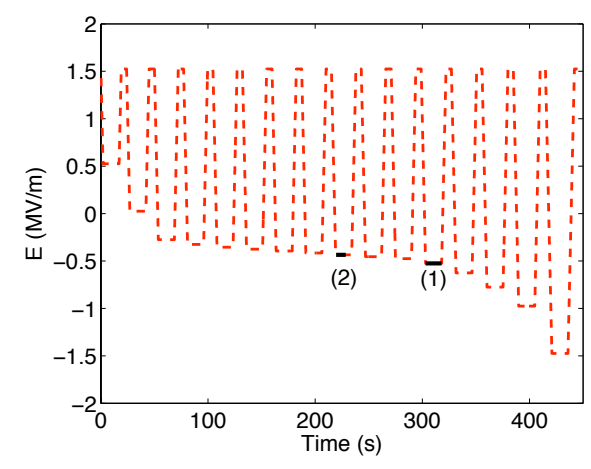

(a)

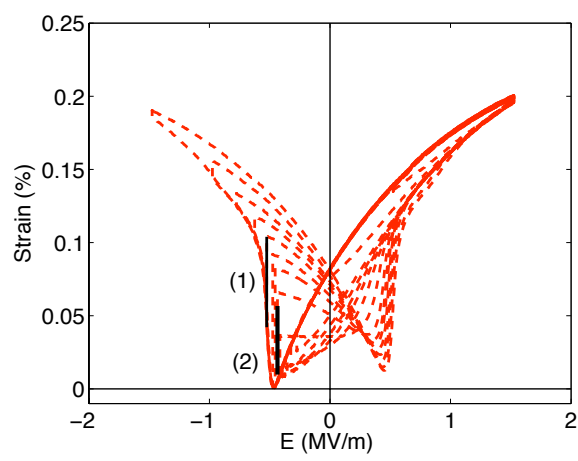

(c)

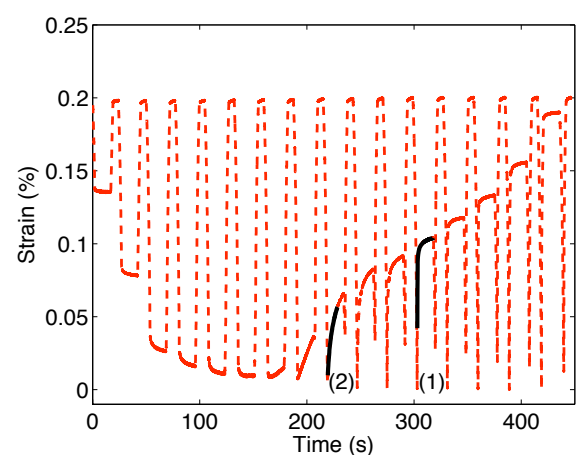

(b)
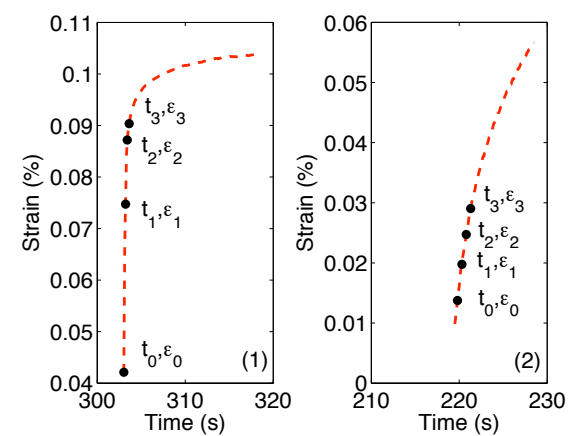

(d)

Figure 6: Zero prestress strain data from [24] used to obtain initial estimates of $\gamma$ and $\tau_{90}$.

for $E>E_{c}$. The expression for $E<-E_{c}$ is identical. From (3), (41) and (42), it follows that

$$
\begin{array}{ll}
\frac{1}{\tau_{90}} e^{-\gamma \Delta G_{90-}^{a}(E)}=\frac{1}{\Delta t} \ln \left(\frac{e_{2}}{e_{3}}\right), \quad E<-E_{c} \\
\frac{1}{\tau_{90}} e^{-\gamma \Delta G_{90+}^{a}(E)}=\frac{1}{\Delta t} \ln \left(\frac{e_{2}}{e_{3}}\right), \quad E>E_{c} .
\end{array}
$$

We note that if $|E|>E_{c}$, as is the case for the data at $\bar{E}_{1}$ indicated in Figure 6 by (1), energy barriers are eliminated so that $\Delta G_{-90}^{a}(E)=\Delta G_{+90}^{a}(E)=0$ which yields

$$
\tau_{90}=\frac{\Delta t}{\ln \left(e_{2} / e_{3}\right)} .
$$

In this case, the decay data at the second fixed field can be used to solve for $\gamma$. If $|E|<E_{c}$ for both fixed fields, or if $E_{c}$ is unknown, one can eliminate $\tau_{90}$ to first solve for $\gamma$. To illustrate for $\bar{E}_{1}, \bar{E}_{2}<0$ and corresponding strain differences $e_{12}, e_{13}$ and $e_{22}, e_{23}$, elimination of $\tau_{90}$ yields the relation

$$
e^{\gamma \Delta G_{-90}^{a}\left(\bar{E}_{1}\right)} \ln \left(e_{12} / e_{13}\right)=e^{\gamma \Delta G_{-90}^{a}\left(\bar{E}_{2}\right)} \ln \left(e_{22} / e_{23}\right)
$$

for $\gamma$. Note that (44) can be easily solved for $\gamma$ by plotting the solution as a function of $\gamma_{2}$ and approximating the root or by using a symbolic routine; e.g., the symbolic MATLAB command solve.m. The parameter $\tau_{90}$ is then determined from (43). An initial value of $\tau_{180}$ is then taken to be $\tau_{180}=\frac{1}{10} \tau_{90}$. 
The data-driven algorithm to determine initial parameter estimates for the $180^{\circ}$ switching polarization model (16) is analogous.

\section{Initial Estimates for $\tau_{90}$ Based on Frequency-Dependent Data}

Frequency-dependent strain or polarization data, such as that plotted in Figures 4 and 7, can be used in a similar manner to obtain an initial estimate for $\tau_{90}$ or $\tau_{180}$. We let $t_{0}$ denote the time at which a field reversal occurs at $E_{\max }$ or $E_{\text {min. }}$. As before, we let $\varepsilon_{1}, \varepsilon_{2}$ and $\varepsilon_{3}$ designate measured strains at equally spaced subsequent times $t_{1}=t_{0}+\Delta t, t_{2}=t_{0}+2 \Delta t$ and $t_{3}=t_{0}+3 \Delta t$, and let $e_{2}=\varepsilon_{2}-\varepsilon_{1}, e_{3}=\varepsilon_{3}-\varepsilon_{2}$.

Assumption 2. For a field reversal at $E_{\max }$, we make the simplifying assumption that $x_{-} \approx 0$ and that dipole kinetics are dominated by switching from $90^{\circ}$ to $180^{\circ}$ orientations; i.e., $p_{90-}=$ $p_{+90}=p_{+-}=0$. Similarly, for reversal at $E_{\text {min }}$, we assume that $x_{+} \approx 0$ and $p_{-90}=p_{90+}=$

$p_{-+}=0$. These are the same assumptions made in Assumption 1. Finally, we assume that activation energies are sufficiently small to permit the approximation

$$
p_{90+}=p_{90-}=\frac{1}{\tau_{90}} .
$$

With these assumptions, (11) reduces to

$$
\begin{aligned}
& \dot{x}_{-}=\frac{-1}{\tau_{90}} x_{-}+\frac{1}{\tau_{90}} \quad, \quad E \approx E_{\min } \\
& \dot{x}_{+}=\frac{-1}{\tau_{90}} x_{+}+\frac{1}{\tau_{90}} \quad, \quad E \approx E_{\max }
\end{aligned}
$$

which has the solution

$$
\begin{array}{ll}
x_{-}(t, E)=1-\left(x_{-}\left(t_{0}, E\right)-1\right) e^{-\left(t-t_{0}\right) / \tau_{90}} & , \quad E \approx E_{\min } \\
x_{+}(t, E)=1-\left(x_{+}\left(t_{0}, E\right)-1\right) e^{-\left(t-t_{0}\right) / \tau_{90}} & , \quad E \approx E_{\max } .
\end{array}
$$

If we assume that $E_{1} d_{+} \approx E_{2} d_{+} \approx E_{3} d_{+} \approx E_{\max }$, analysis similar to that used to obtain (42) yields the relation

$$
\tau_{90}=\frac{\Delta t}{\ln \left(e_{2} / e_{3}\right)}
$$

which provides an initial, data-driven estimate for $\tau_{90}$. Due to the assumption (45), which is more stringent than the case for creep data where fields are fixed, this data does not readily admit a data-driven algorithm to initially estimate $\gamma$. Hence it must simply be estimated through a least squares fit to data.

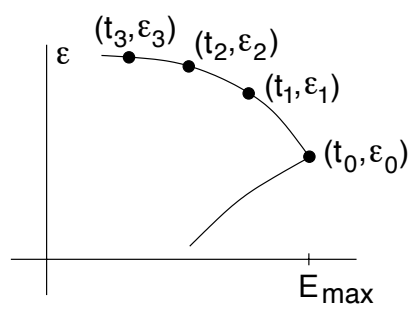

Figure 7: Frequency-dependent strain data used to obtain an initial estimate for $\tau_{90}$. 


\section{Model Calibration and Validation for $\mathrm{PZT}$ and $\mathrm{BaTiO}_{3}$}

We illustrate the performance of the data-driven parameter estimation algorithms using PZT data reported in [24] and $\mathrm{BaTiO}_{3}$ data from [5].

For the PZT data, polarization and strain measurements, in the absence of applied mechanical stresses, were collected using a P-802.00 piezo-actuator whereas a prestressed NEC Model AEO505D08 actuator with a restoring spring was used to collect stress-dependent data. Both are multilayer devices with similar dimensions so we briefly summarize the latter and refer the reader to [24] for details regarding both devices.

The AEO505D08 actuator was comprised of 80 active layers, each of thickness $0.1 \mathrm{~mm}$. The dimensions, including packaging, were $6.5 \times 6.5 \times 10 \mathrm{~mm}$ which yielded the cross-sectional area $A=42.25 \mathrm{~mm}^{2}=4.225 \times 10^{-5} \mathrm{~m}^{2}$. The polarization was measured using a Sawyer-Tower circuit comprised of a reference capacitor connected in series with the PZT actuator. Displacements were measured using a fiber-optic displacement sensor having an effective resolution of $0.1 \mu \mathrm{m}$ and strains were computed by dividing the total thickness $(8 \mathrm{~mm})$ of the active layers.

When reporting the accuracy of model fits, we define the relative errors to be

$$
e_{r e l}=\frac{\sum_{i=1}^{N_{d}}\left(w_{P}\left[P\left(E\left(t_{i}\right), \sigma ; q\right)-P_{i}\right]^{2}+w_{\varepsilon}\left[\varepsilon\left(E\left(t_{i}\right), \sigma ; q\right)-\varepsilon_{i}\right]^{2}\right)}{\sum_{i=1}^{N_{d}}\left(w_{P} P_{i}^{2}+w_{\varepsilon} \varepsilon_{i}^{2}\right)} .
$$

The functionals (34) and (35), with the analytic Jacobians constructed using the algorithms in Section 4.1, were minimized using the MATLAB nonlinear least squares routine 1sqnonlin with the options 'Algorithm'='trust-region-reflective', 'TolFun' $=1 e-4$, 'Jacobian' $=$ 'on' and a lower bound of 0 specified for all parameters except $\varepsilon_{R}^{90}$ which was bounded below by $-1 \times 10^{-2}$.

\subsection{Zero Prestress PZT Data}

\section{$90^{\circ}$ Polarization-Strain Model}

We first illustrate the calibration and performance of the $90^{\circ}$ switching polarization-strain model (8) and $180^{\circ}$ switching polarization model (16) using zero prestress data reported in [24] obtained using a P-802.00 PZT actuator.

The data used to obtain initial parameter values is plotted in Figures 6 and 8 and resulting values, obtained using the algorithms in Table 4 are compiled in Table 5. Corresponding model predictions and initial density representations are respectively plotted in Figure 9 and Figure 11(a) and (c). The
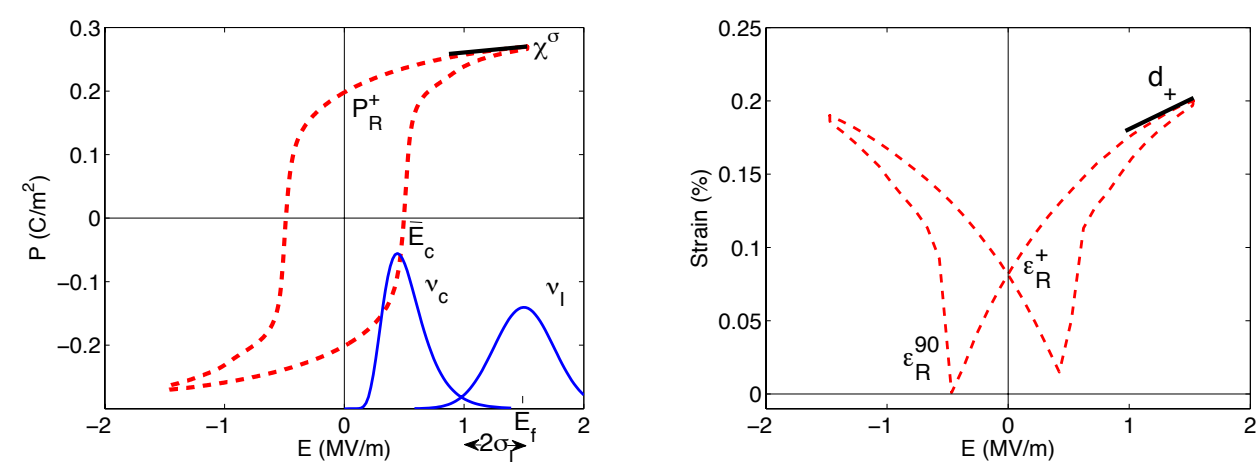

Figure 8: Zero prestress polarization and strain data from [24] used to obtain initial estimates of $P_{R}^{+}, \varepsilon_{R}^{+}, \varepsilon_{R}^{90}, \chi^{\sigma}, \bar{F}_{c}, \sigma_{c}$ and $\sigma_{I}$. 

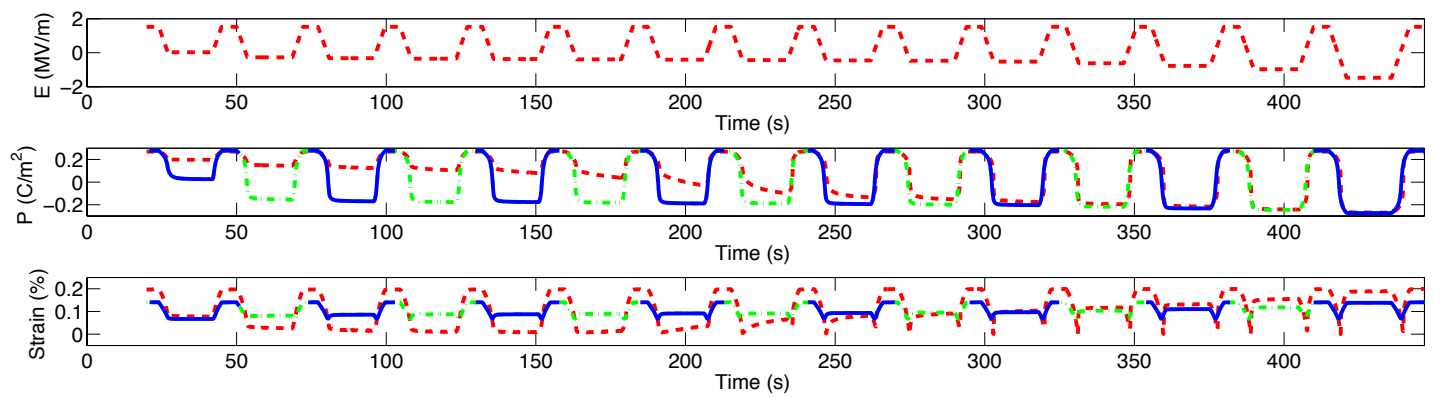

(a)

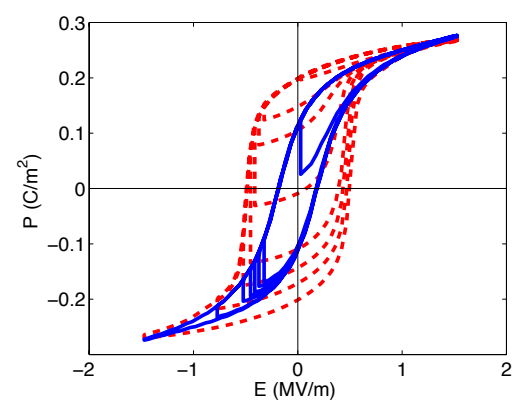

(b)

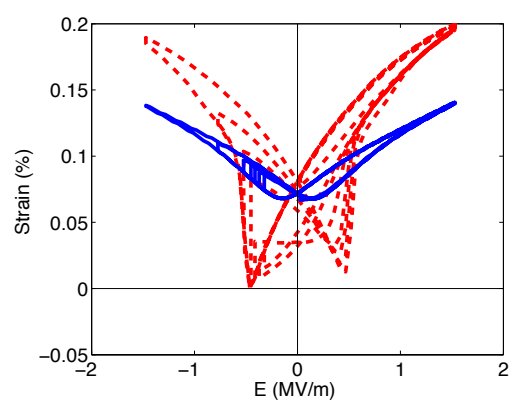

(c)

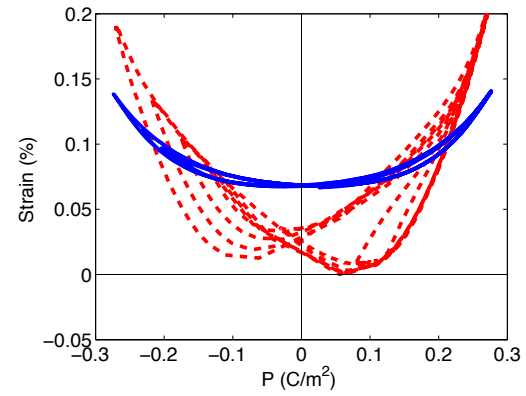

(d)

Figure 9: Polarization and strain model prediction to zero-prestress data from [24] using initial parameter values obtained using the data-driven algorithms in Table 4: (a) time domain and (b)-(d) phase space; (- - $)$ experimental data, $(-\bullet-\bullet)$ model fit, $(-(-)$ model fits for 8 selected loading cycles. Labels (1) and (2) in Figure 6 indicate strain data used to find initial estimates for $\gamma$ and $\tau_{90}$.

initial values of $P_{R}^{+}, \varepsilon_{R}^{+}, \varepsilon_{R}^{90}$ and $\chi^{\sigma}$ are obtained directly from the polarization and stain data shown in Figure 8. The density coefficient $\bar{F}_{c}=P_{R}^{+} \bar{E}_{c}$ results from $\bar{E}_{c}=0.5 \times 10^{6}$, and $2 \sigma_{I}=0.5 \times 10^{6}$ yields $\sigma_{I}=0.25 \times 10^{6}$. Similarly, the relations in Figure 5 yield $\sigma_{c}=0.5 \log ((1.5-0.5) / 0.5) \approx 0.35$. Finally, the initial estimates for $\gamma$ and $\tau_{90}$ were computed using the data shown in Figure 6 . The initial value $d_{+}=374 \times 10^{-12}$ is a literature value of $d_{33}$ reported for PZT.

Whereas the model quantifies the trends exhibited by the data, the initial predictions are not sufficiently accurate for material or device characterization. We then minimized the functional (35) using 2140 data values to obtain the optimized parameters reported in Table 5 and the resulting fits and densities shown in Figure 10 and 11(b) and (d). The optimized relative error is 10.99\% It is observed that the calibrated polarization and strain models accurately fit the data in all drive regimes including the saturation and burst regions, the creep regions, and minor loops. Moreover, use of the analytic Jacobian and efficient implementation algorithms permitted the optimization to

\begin{tabular}{|c|c|c|c|c|c|c|c|c|}
\hline & $P_{R}^{+}$ & $\varepsilon_{R}^{+}$ & $\varepsilon_{R}^{90}$ & $\chi_{+}^{\sigma}$ & $d_{+}$ & $\gamma$ & $\tau_{90}$ & $\tau_{180}$ \\
\hline Init. & 0.2 & $0.081 \times 10^{-2}$ & 0.0 & $4.71 \times 10^{-8}$ & $374 \times 10^{-12}$ & $1.38 \times 10^{-4}$ & 0.64 & .064 \\
\hline Opt. & 0.18 & $0.050 \times 10^{-2}$ & $-5.64 \times 10^{-2}$ & $5.35 \times 10^{-8}$ & $930.46 \times 10^{-12}$ & $2.08 \times 10^{-3}$ & 0.41 & .043 \\
\hline
\end{tabular}

Table 5: Initial and optimized parameter values for the polarization and strain model with zero prestress. The fixed density parameters were $\bar{F}_{c}=0.2 \times 10^{6}, \sigma_{I}=0.25 \times 10^{6}$ and $\sigma_{c}=0.35$. The optimized density parameters $\alpha_{1}=1.65, \alpha_{0}=0.92, \alpha_{-1}=0.77, \alpha_{-2}=2.55, \alpha_{-3}=6.69$ and $\beta_{1}=0.79, \beta_{0}=1.24, \beta_{-1}=1.12, \beta_{-2}=2.95, \beta_{-3}=9.45$ were obtained using initial estimates of 1 . 

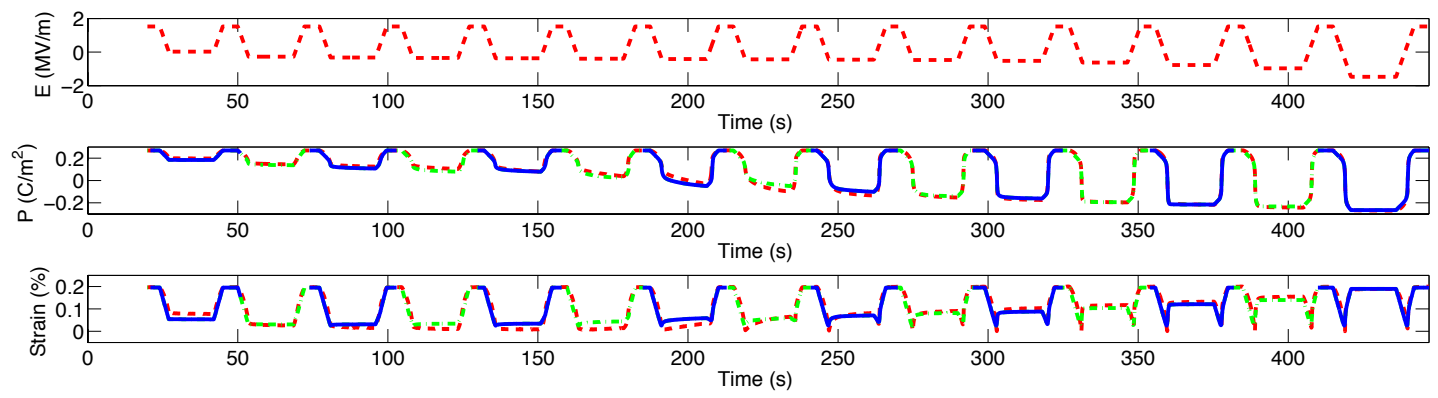

(a)

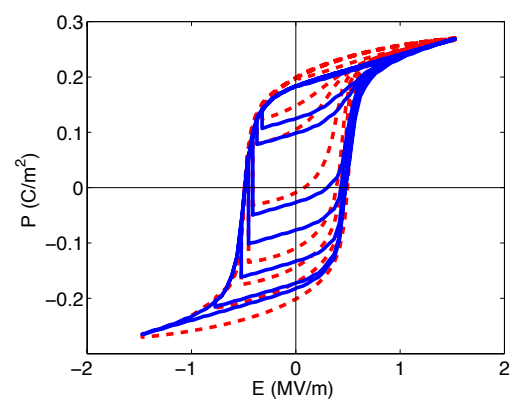

(b)

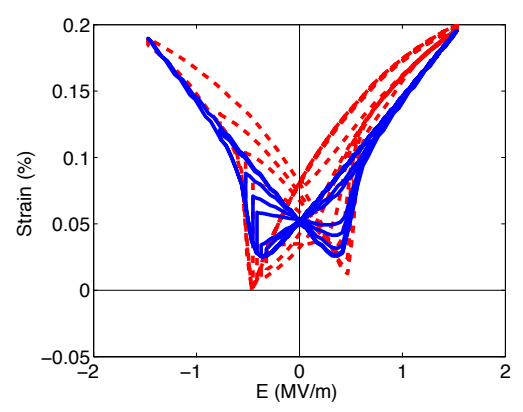

(c)

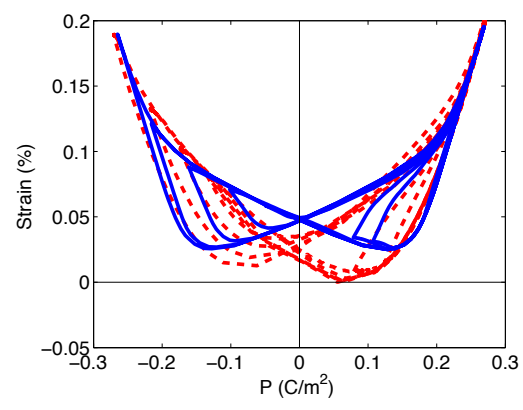

(d)

Figure 10: Optimized fit of the polarization and strain model to zero-prestress data from [24]: (a) time domain and (b)-(d) phase space; $\left(--_{-}\right)$experimental data, $(-\bullet-\bullet)$ model fit, $(-\longrightarrow)$ model fits for 8 selected loading cycles.

be completed in $673 \mathrm{~s}$ on a Mac Pro with a $2.26 \mathrm{GHz}$ processor and 8 GB of memory.

We note that the final parameter values in Table 5 are quite close to the initial values predicted by the data-driven algorithms. Hence much of the inaccuracy in the initial model prediction is due to the initial density parameter choices $\alpha_{k}=\beta_{k}=1$. The initial values of $\alpha_{k}$ and $\beta_{k}$ can be easily tuned to provide more accurate fits; however, the choice of 1 simplifies the data-driven algorithms and is sufficiently accurate so that the optimization routine yields accurate fits in a timely manner.

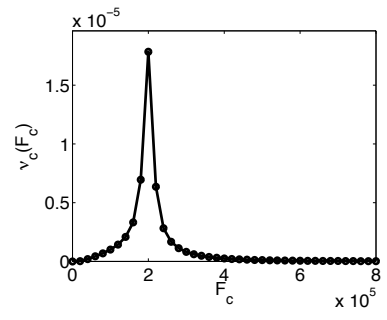

(a)

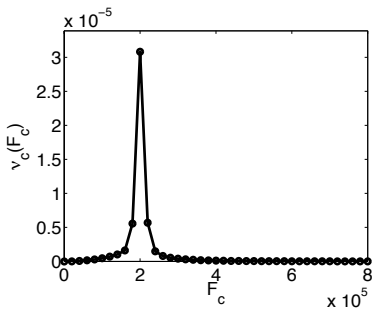

(b)

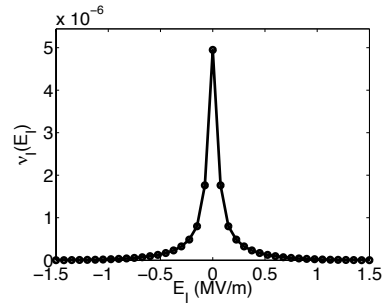

(c)

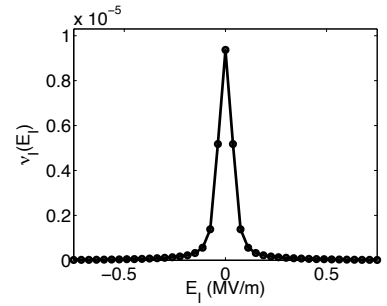

(d)

Figure 11: (a) Initial and (b) optimized critical driving force density $\nu_{c}\left(F_{c}\right)$ with 41 equally spaced quadrature points marked as dots. (c) Initial and (d) optimized interaction field density $\nu_{I}\left(E_{I}\right)$.

\section{$180^{\circ}$ Polarization Model}

For applications and operating regimes with negligible to moderate prestresses $\sigma_{0}$, the fieldpolarization response exhibits minimal dependency on $90^{\circ}$ switching. In these cases, the polarization can be adequately modeled using the $180^{\circ}$ switching model summarized in Table 2. 


\begin{tabular}{|c|c|c|c|c|}
\hline & $P_{R}$ & $\eta^{\sigma}$ & $\gamma$ & $\tau_{90}$ \\
\hline Init. & 0.2 & $0.25 \times 10^{8}$ & $2.79 \times 10^{-3}$ & 0.15 \\
\hline Opt. & 0.20 & $0.22 \times 10^{8}$ & $3.78 \times 10^{-3}$ & 0.10 \\
\hline
\end{tabular}

Table 6: Initial and optimized parameter values for the $180^{\circ}$ model zero prestress.

To illustrate, we employed the data-driven algorithms of Section 4.2 to obtain the parameter estimates compiled in Table 6. We then minimized the functional (34) using 2140 data points to obtain the optimized fit and densities shown in Figure 12. The accuracy of the fit demonstrates the applicability of the $180^{\circ}$ switching model for characterizing the polarization in low to moderate stress regimes. Moreover, the use of the implementation algorithms of Section 3 and analytic Jacobian relations of Section 4.1 renders the model calibration codes highly efficient and optimization was achieved in approximately $36 \mathrm{~s}$ on the Mac Pro.

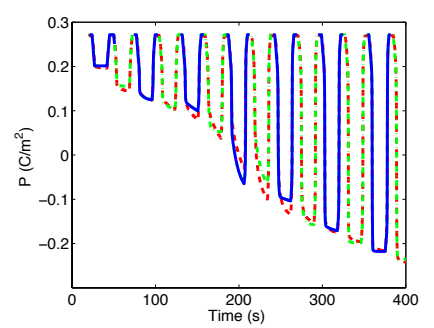

(a)

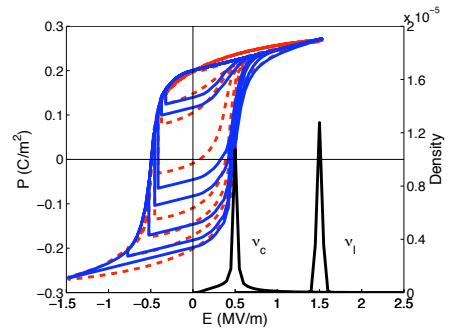

(b)

Figure 12: Optimized fit of the $180^{\circ}$ polarization model to zero-prestress data from [24]: (a) time domain and (b) phase space; (- - ) experimental data, $\left(-\bullet \bullet^{\bullet}\right)$ model fit, (_ $(-)$ model fits for 8 selected loading cycles.

\subsection{Prestressed Actuator}

The estimation of parameters and performance of the polarization-strain model for a prestressed actuator is illustrated in [19] using data reported in [24].

\subsection{Variable Loading Rates}

To illustrate the physical phenomena, and resulting model response, associated with variable loading rates, we consider data from [24] that was collected at loading rates of $5 \mathrm{~V} / \mathrm{s}, 50 \mathrm{~V} / \mathrm{s}$ and $500 \mathrm{~V} / \mathrm{s}$. Since this data was collected using the P-802.00 actuator, that had purely electrical loading, the polarization and strains were modeled using the relations (8) that neglect the prestress $\sigma_{0}$ and spring and damping coefficients $k$ and $c$ associated with restoring mechanisms.

The strain and polarization data plotted in Figure 13 exhibit differing dynamics at the three loading rates, especially in the burst region where $+180^{\circ}$ to $90^{\circ}$ and $90^{\circ}$ to $-180^{\circ}$ dipole transitions are dominant. In the field-polarization plots, this is reflected by delayed first-order minor loops as indicated by the curves (1)-(3). The slower $90^{\circ}$ to $-180^{\circ}$ transitions, and resulting lagging effects at high loading rates, are even more noticeable in the field-strain plots due to the degree to which stains depend on $90^{\circ}$ dipole switching.

To model this rate-dependent phenomena, the data-driven techniques were used to compute the initial parameter estimates compiled in Table 7 along with the density coefficients $\bar{F}_{c}=0.2 \times 10^{6}, \sigma_{c}=$ 0.347 and $\sigma_{I}=0.25 \times 10^{6}$. We then minimized the functional (43), using the $5 \mathrm{~V} / \mathrm{s}$ and $500 \mathrm{~V} / \mathrm{s}$ 


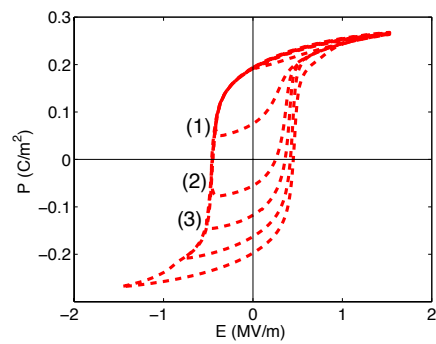

(a)

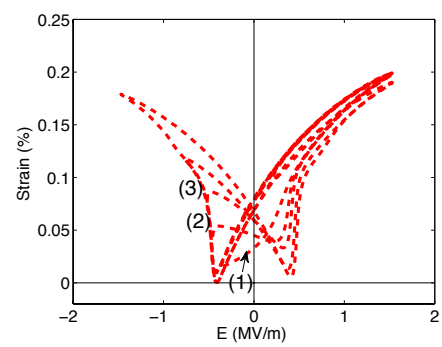

(d)

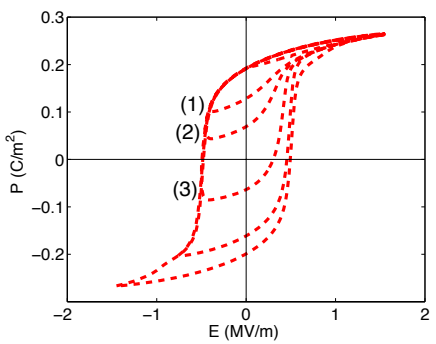

(b)

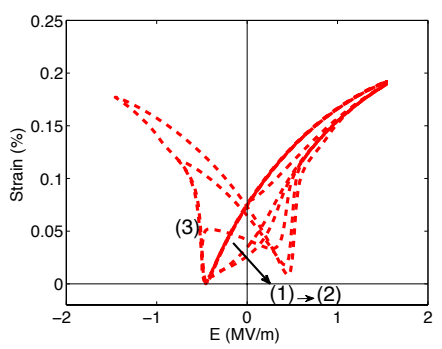

(e)

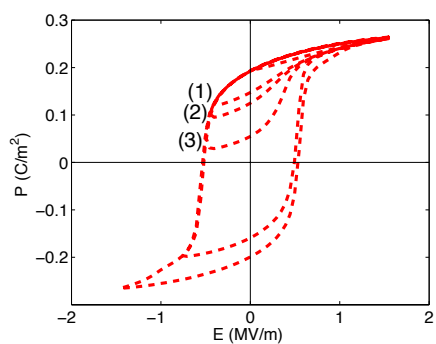

(c)

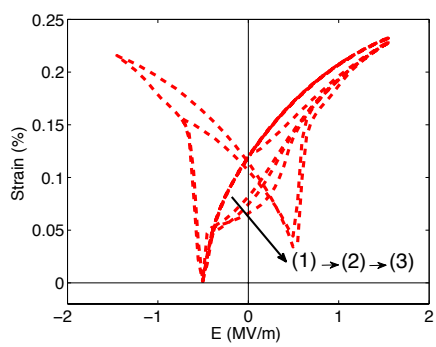

(f)

Figure 13: Polarization and strain data from [24], collected at (a),(d) $5 \mathrm{~V} / \mathrm{s}$, (b), (e) $50 \mathrm{~V} / \mathrm{s}$, and (c),(f) $500 \mathrm{~V} / \mathrm{s}$.

data, with $w_{P}=1, w_{\varepsilon}=40,000$, to obtain the optimized parameters in Table 7 , the densities shown in Figure 14, and the model fits plotted in Figure 12. We note that the model is predicting the $50 \mathrm{~V} / \mathrm{s}$ behavior.

The time-polarization and time-strain plots illustrate that the model is quantifying both the major and minor loop behavior at the three loading rates. The phase plots illustrate that while the model is very accurately characterizing the field-polarization behavior, there is some inaccuracy in the field-strain fits and prediction. This may be due to limitations in the relations used to quantify $90^{\circ}$ and $180^{\circ}$ switching mechanisms. However, the model is quantifying the lagged switching behavior at $500 \mathrm{~V} / \mathrm{s}$ that is present in both the polarization and strain data. We note that improved fits for each loading rate can be achieved if parameters are optimized for that rate. The dynamic nature of the model is illustrated by the fact that it adequately characterizes the rate-dependent material behavior using one set of parameters. This makes it advantageous for material characterization, device optimization, and control design of applications requiring variable loading rates.

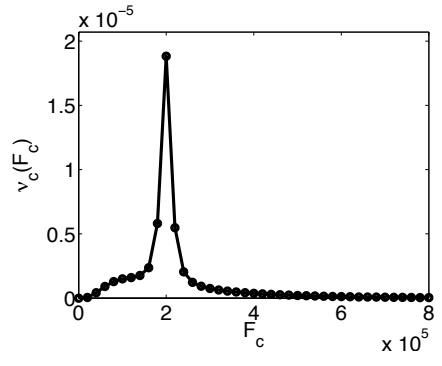

(a)

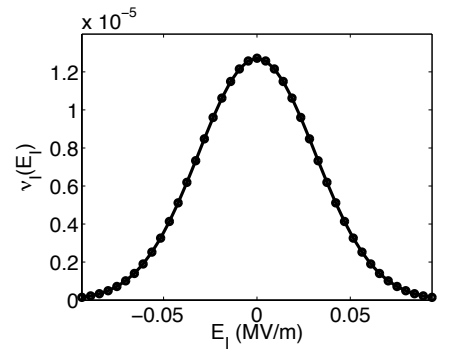

(b)

Figure 14: (a) Critical driving force density $\nu_{c}\left(F_{c}\right)$ and (b) interaction field density $\nu_{I}\left(E_{I}\right)$ with 41 equally spaced quadrature points marked as dots. 

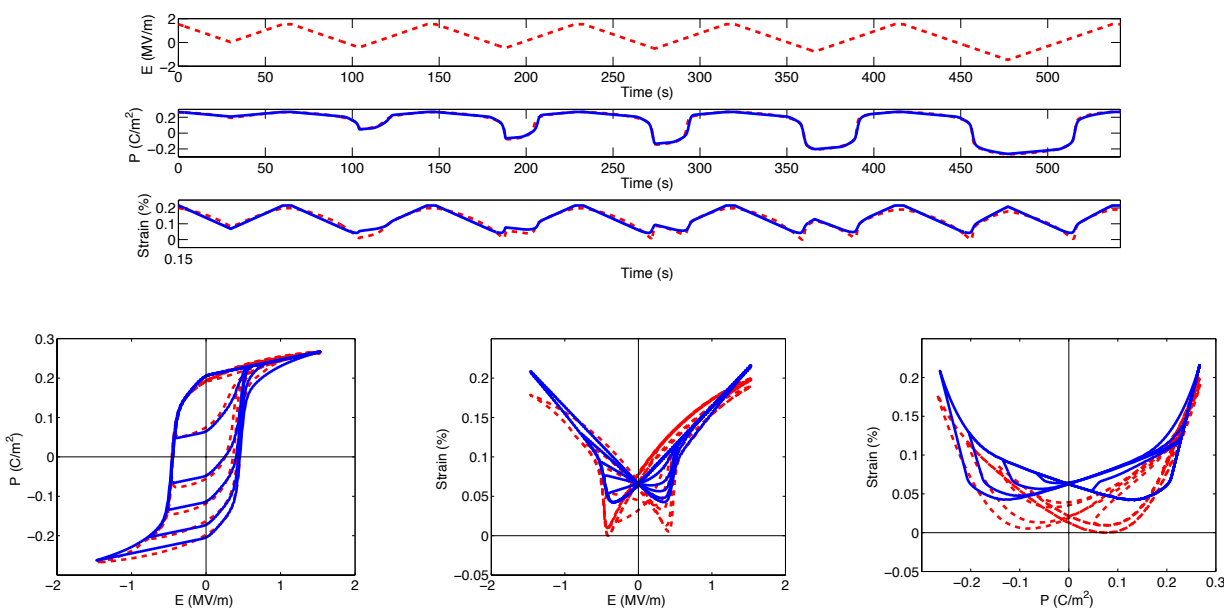

(a)
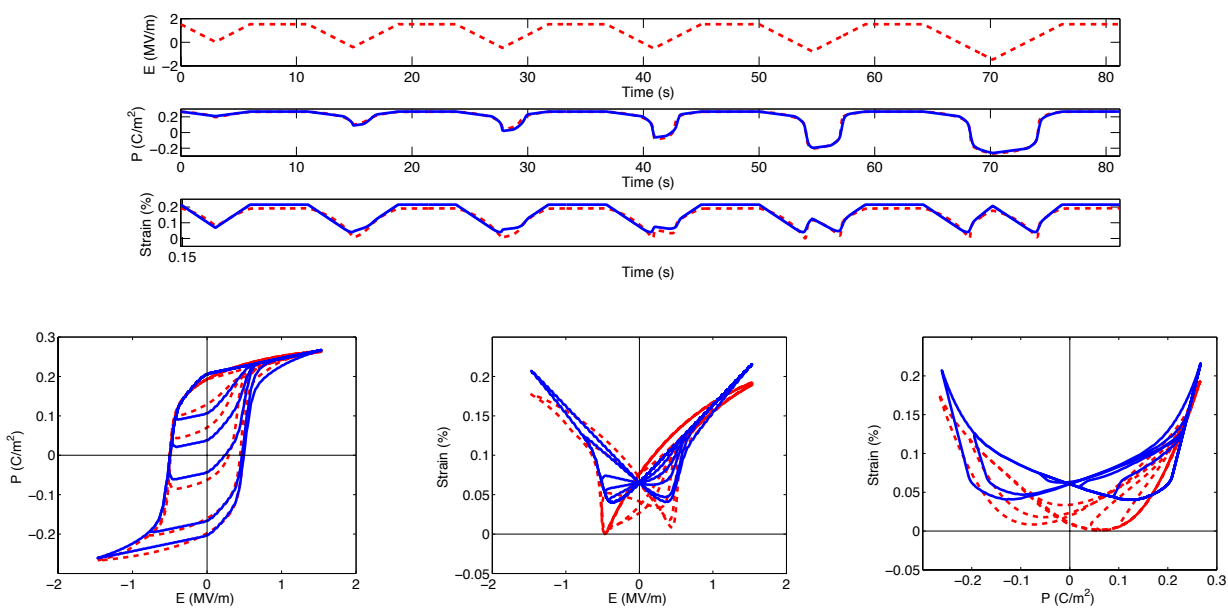

(b)
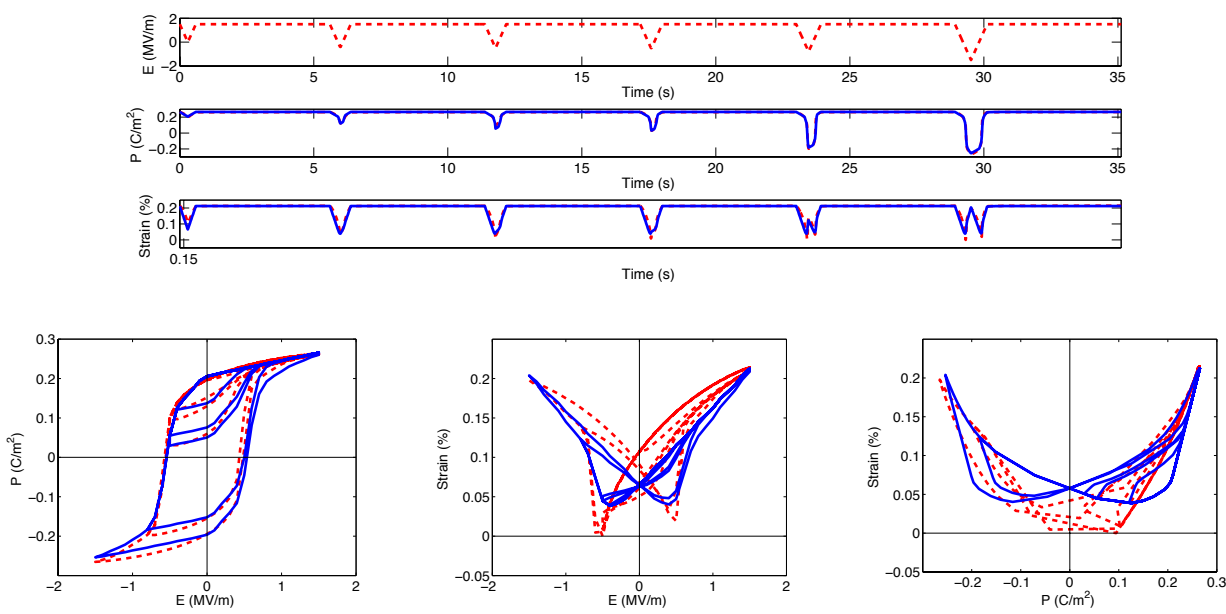

(c)

Figure 15: Optimized fit of the polarization-strain model to data from [24] for loading rates of (a) $5 \mathrm{~V} / \mathrm{s}$ and (c) $500 \mathrm{~V} / \mathrm{s}$. (b) Model prediction for a loading rate of $50 \mathrm{~V} / \mathrm{s}$ : (- - -) experimental data, (—) model fit. 


\begin{tabular}{|c|c|c|c|c|c|c|c|c|}
\hline & $P_{R}^{+}$ & $\varepsilon_{R}^{+}$ & $\varepsilon_{R}^{90}$ & $\chi_{+}^{\sigma}$ & $d_{+}$ & $\gamma$ & $\tau_{90}$ & $\tau_{180}$ \\
\hline Init & 0.18 & $0.050 \times 10^{-2}$ & $-5.64 \times 10^{-2}$ & $5.35 \times 10^{-8}$ & $930.46 \times 10^{-12}$ & $2.08 \times 10^{-3}$ & 0.41 & 0.043 \\
\hline Opt & 0.21 & $0.066 \times 10^{-2}$ & $-0.071 \times 10^{-2}$ & $3.93 \times 10^{-8}$ & $992.26 \times 10^{-12}$ & $8.37 \times 10^{-3}$ & $1.48 \times 10^{-9}$ & 0.16 \\
\hline
\end{tabular}

Table 7: Initial and optimized parameter values for the polarization and strain model obtained using loading rates of $5 \mathrm{~V} / \mathrm{s}$ and $500 \mathrm{~V} / \mathrm{s}$. The optimized density parameters are $\alpha_{1}=5.02, \alpha_{0}=$ $1.82 \times 10^{-2}, \alpha_{-1}=1.24, \alpha_{-2}=2.56, \alpha_{-3}=2.89$ and $\beta_{1}=4.63 \times 10^{-2}, \beta_{0}=1.13 \times 10^{-8}, \beta_{-1}=$ $4.71 \times 10^{-8}, \beta_{-2}=3.42 \times 10^{-14}, \beta_{-3}=11.01$.

\subsection{Single Crystal $\mathrm{BaTiO}_{3}$}

Here we illustrate the performance of the model for characterizing the behavior of single crystal $\mathrm{BaTiO}_{3}$ using data from [5]. For the data considered here, the applied stress was parallel to the applied field thus motivating consideration of a uniaxial model. Furthermore, we employ the grainlevel or single crystal relations (10).

We first determined the initial and optimized parameter values reported in Table 8 through a fit to the data collected at prestresses of $-0.36 \mathrm{MPa}$ and $-1.78 \mathrm{MPa}$. The correlation of the parameter values for $P_{R}^{+}, \varepsilon_{R}^{+}, \varepsilon_{R}^{90}, \chi_{+}^{\sigma}$ and $d_{+}$to corresponding properties of the data can be directly observed. We also note that $\tau_{90}>\tau_{180}$ which indicates that $180^{\circ}$ switching occurs more quickly than $90^{\circ}$ switching. This is consistent with analogous observations for PZT, reported in [23], and explains the flat behavior of the $P-\varepsilon$ data.

The resulting fit at $-0.36 \mathrm{MPa}$ and $-1.78 \mathrm{MPa}$ and predictions at prestresses of $-0.72 \mathrm{MPa}$ and $0 \mathrm{MPa}$ are shown in Figure 16. It is observed that the polarization and strain fits at $-0.36 \mathrm{MPa}$ and $-1.78 \mathrm{MPa}$ are quite accurate as are the polarization predictions at the other two prestresses. Whereas the strain prediction at $-0.72 \mathrm{MPa}$ is moderately accurate, the model overpredicts the strains for the unstressed crystal where $90^{\circ}$ switching is minimal. This is in accordance with the results reported in [12] and indicates some limitations in the $90^{\circ}$ switching characterization in the absence of prestresses.

\begin{tabular}{|c|c|c|c|c|c|c|c|c|c|}
\hline & $P_{R}^{+}$ & $\varepsilon_{R}^{+}$ & $\varepsilon_{R}^{90}$ & $\chi_{+}^{\sigma}$ & $d_{+}$ & $s^{E}$ & $\gamma$ & $\tau_{90}$ & $\tau_{180}$ \\
\hline Init & 0.26 & $0.67 \times 10^{-2}$ & $-0.12 \times 10^{-2}$ & $1.5 \times 10^{-8}$ & $85.6 \times 10^{-12}$ & $15.7 \times 10^{-12}$ & 0.015 & 0.06 & 0.01 \\
\hline Opt & 0.25 & $0.73 \times 10^{-2}$ & $-0.99 \times 10^{-2}$ & $2.82 \times 10^{-8}$ & $841.89 \times 10^{-12}$ & $26.83 \times 10^{-11}$ & 0.00041 & 0.34 & 0.00041 \\
\hline
\end{tabular}

Table 8: Initial and optimized $\mathrm{BaTiO}_{3}$ parameter values obtained through a fit to $-0.36 \mathrm{MPa}$ and $-1.78 \mathrm{MPa}$ prestressed data from [5]. The density representation (14) employed the optimized value $\bar{F}_{c}=0.0145 \times 10^{6}$.

\section{Concluding Remarks}

The homogenized energy model is a multiscale, micromechanical framework that quantifies hysteresis and constitutive nonlinearities intrinsic to ferroelectric materials. It incorporates mechanisms at multiple spatial scales by combining energy analysis at the domain level with stochastic homogenization techniques to construct material-level constitutive relations. The multiple timescales associated with dipole switching processes are incorporated by employing Boltzmann principles to quantify transition rates associated with dipole fractions. In combination, the framework characterizes a range of rate-dependent hysteretic phenomena associated with the materials. Furthermore, constitutive 


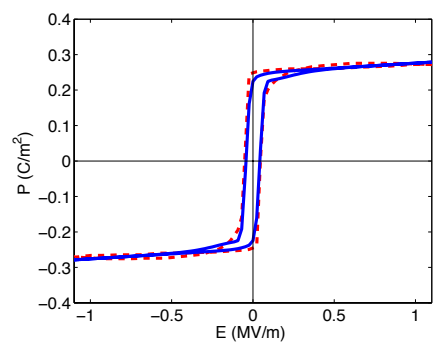

(a)

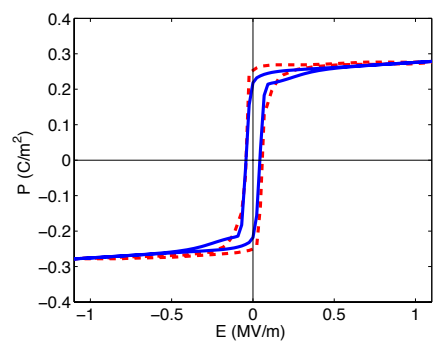

(d)

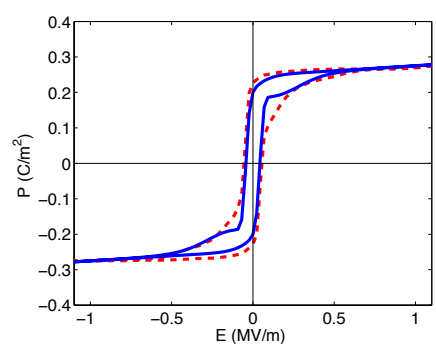

(g)

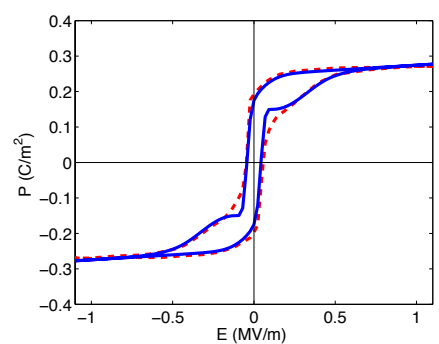

(j)

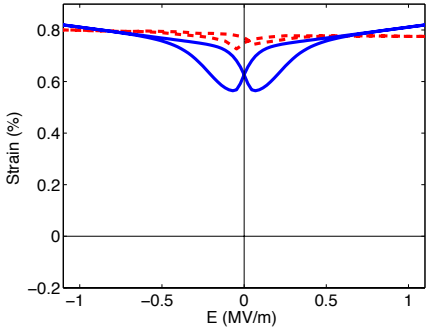

(b)

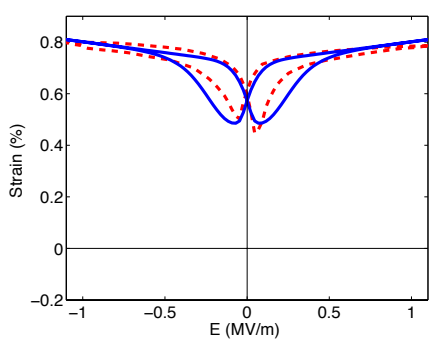

(e)

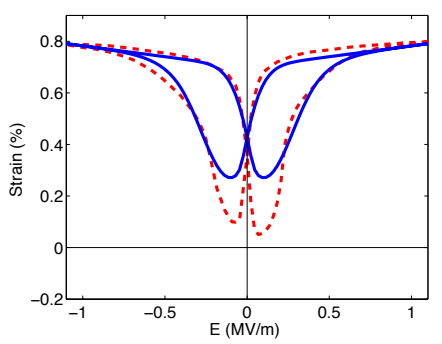

(h)

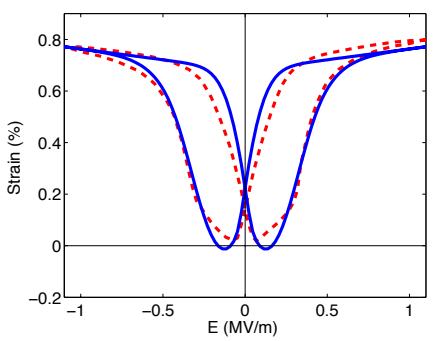

(k)

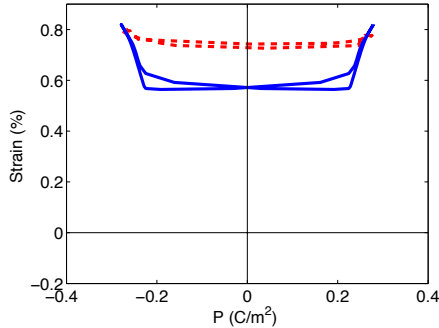

(c)

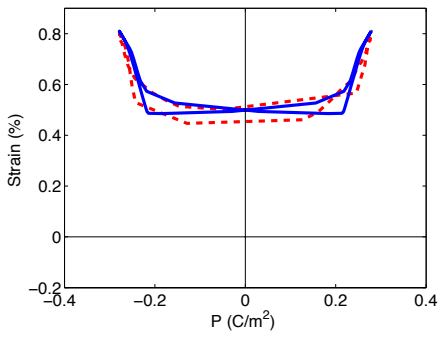

(f)

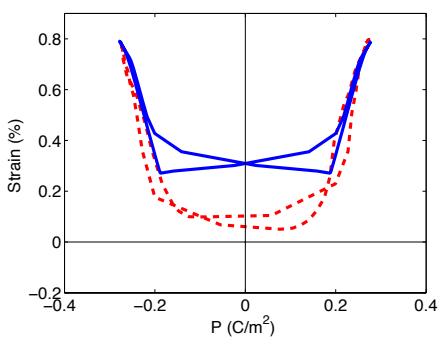

(i)

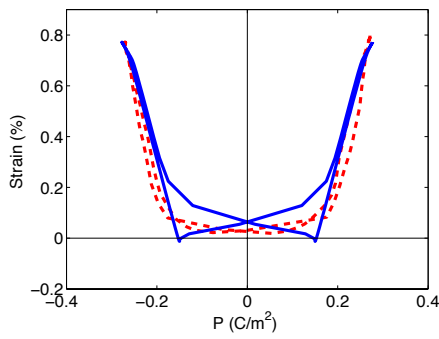

$(\ell)$

Figure 16: Fit of the polarization and strain model to single crystal $\mathrm{BaTiO}_{3}$ data from [5] with a prestress of (d)-(f) -0.36 MPa and (j)- $(\ell)-1.78 \mathrm{MPa}$. Model predictions for prestresses of (a)-(c) $0 \mathrm{MPa}$ and $(\mathrm{g})-(\mathrm{i})-1.07 \mathrm{Mpa}$ ( - - $\left._{-}\right)$experimental data, (__ $)$model fit.

relations resulting from the framework can be directly employed to construct distributed models, for complex devices, that are amenable to finite element implementation.

In this paper, we presented algorithms that facilitate efficient model calibration and implementation. The efficiency of the parameter estimation algorithms is based in part on the physical nature of the model which permits model parameters to be correlated with properties of field-polarization and field-strain data as shown in Figure 4. Specifically, we presented data-driven algorithms to determine initial estimates for $P_{R}^{+}, \varepsilon_{R}^{+}, \varepsilon_{R}^{90}, \chi^{\sigma}, d_{+}, \gamma, \tau_{90}, \tau_{180}$ that facilitate subsequent optimization through least squares fits to the data. Furthermore, this data can be used to determine values for 
the density coefficients, $\bar{E}_{c}, \bar{F}_{c}, \sigma_{c}$ and $\sigma_{I}$.

Major loop, biased minor loop, and creep data, of the form depicted in Figure 4, represents ideal data to be collected if there is flexibility regarding the design of characterization experiments. However, in many cases, one is limited to biased minor loop data such as that shown in Figure 1. In such cases, one can employ values previously reported in the literature as initial values for optimization routines.

The coefficients $d_{33}$ and $s^{E}$ and relative permittivity $\epsilon_{r}$, which can be used to compute $\chi_{\sigma}$, are commonly reported for ferroelectric materials and documented values can be employed as initial parameter estimates. The remanence polarization $P_{R}$, remanence strain $\varepsilon_{R}$, and coercive field $E_{c}$ are also fundamental properties of ferroelectric materials that are often reported only in material characterization investigations rather than in compilations for transducer design. The model parameters $P_{R}^{+}, \varepsilon_{R}^{+}, \varepsilon_{R}^{90}, \gamma, \tau_{90}$ and $\tau_{180}$ are related to intrinsic material properties that are critical to actuator and sensor design and characterization.

This motivates the compilation of material property and parameter tables and libraries that facilitate the calibration of models for devices that employ these materials. Representative values of $d_{33}, s_{11}^{E}, \epsilon_{r}, \chi^{\sigma}$ from the literature and $P_{R}, \varepsilon_{R}, E_{c}$ from the data employed here, and in the companion paper [19], are respectively compiled in Tables 9 and 10. Similarly, representative values of $P_{R}^{+}, \varepsilon_{R}^{+}, \varepsilon_{R}^{90}, \gamma, \tau_{90}, \tau_{180}$ for PZT, PLZT and $\mathrm{BaTiO}_{3}$ are summarized in Table 11 . We note that while variations in these parameters exist for differing devices and operating regimes, these representative values can be employed to initialize optimization routines. Furthermore, it is noted in Appendix A that we are developing a website of data libraries, simulation codes, and modeling frameworks for ferroelectric, ferromagnetic, and ferroelastic materials. Data properties and parameters that can be used for material and device characterization will be compiled and updated at this site.

\begin{tabular}{|c|c|c|c|c|}
\hline & $d_{33}(\mathrm{pC} / \mathrm{N})$ & $s_{11}^{E}\left(\mathrm{~m}^{2} / \mathrm{N}\right)$ & $\epsilon_{r}(\mathrm{C} / \mathrm{Vm})$ & $\chi^{\sigma}=\epsilon_{0}\left(\epsilon_{r}-1\right)(\mathrm{C} / \mathrm{Vm})$ \\
\hline $\mathrm{PZT} 5 \mathrm{~A}$ & 374 & $1.88 \times 10^{-11}$ & 1800 & $1.59 \times 10^{-8}$ \\
$\mathrm{BaTiO}_{3}$ & 85.6 & $8.05 \times 10^{-12}$ & 163 & $1.47 \times 10^{-9}$ \\
$\mathrm{PLZT}$ & 1188 & $1.47 \times 10^{-11}$ & 6356 & $2.99 \times 10^{-8}$ \\
\hline
\end{tabular}

Table 9: Material properties for PZT-5A [17], $\mathrm{BaTiO}_{3}$ [10] and PLZT [14]. Note that the free space permittivity has the value $\epsilon_{0}=8.85 \times 10^{-12}$.

\begin{tabular}{|c|c|c|c|}
\hline & $P_{R}\left(\mathrm{C} / \mathrm{m}^{2}\right)$ & $\varepsilon_{R}$ (Unitless) & $E_{c}(\mathrm{MV} / \mathrm{m})$ \\
\hline $\mathrm{PZT}-5 \mathrm{~A}$ & 0.2 & $0.081 \times 10^{-2}$ & 0.5 \\
$\mathrm{BaTiO}_{3}$ & 0.26 & $0.67 \times 10^{-2}$ & 0.05 \\
$\mathrm{PLZT}$ & 0.25 & $0.25 \times 10^{-2}$ & 0.36 \\
\hline
\end{tabular}

Table 10: Remanence polarizations, remanence strains, and coercive fields for the PZT data [24], single crystal $\mathrm{BaTiO}_{3}$ data [5] and PLZT data [14].

\section{A Available Codes}

To facilitate model validation and dissemination to the community, we have made codes and data available at the website http://www4.ncsu.edu/ jhcrews/smart/code/pzt/index.html. 


\begin{tabular}{|c|c|c|c|c|c|c|c|c|}
\hline & $P_{R}^{+}$ & $\varepsilon_{R}^{+}$ & $\varepsilon_{R}^{90}$ & $\chi_{+}^{\sigma}$ & $d_{+}$ & $\gamma$ & $\tau_{90}$ & $\tau_{180}$ \\
\hline $\mathrm{PZT}$ & 0.18 & $0.050 \times 10^{-2}$ & $-5.64 \times 10^{-2}$ & $5.35 \times 10^{-8}$ & $930.46 \times 10^{-12}$ & $2.08 \times 10^{-3}$ & 0.41 & 0.043 \\
$\mathrm{BaTiO}_{3}$ & 0.25 & $0.73 \times 10^{-2}$ & $-0.99 \times 10^{-2}$ & $2.82 \times 10^{-8}$ & $841.89 \times 10^{-12}$ & $4.1 \times 10^{-4}$ & 0.34 & 0.00041 \\
$\mathrm{PLZT}$ & 0.24 & $0.13 \times 10^{-2}$ & $-0.03 \times 10^{-2}$ & $10.06 \times 10^{-8}$ & $1390.5 \times 10^{-12}$ & $9.62 \times 10^{-4}$ & 0.032 & 0.70 \\
\hline
\end{tabular}

Table 11: Representative parameter values for $\mathrm{PZT}, \mathrm{BaTiO}_{3}$ and PLZT. Appropriate units are given in the notation table in Section 1.

\section{B Lognormal Density}

We summarize here aspects regarding the lognormal density that are pertinent to constructing coercive field densities. Details can be found in $[1,11]$.

If $Y \sim N(\mu, \sigma)$ is a normal random variable with mean $\mu$ and standard deviation $\sigma, X=\exp (Y)$ is lognormally distributed, $X \sim \ln N(\mu, \sigma)$, with density

$$
f(x ; \mu, \sigma)=\frac{1}{x \sigma \sqrt{2 \pi}} e^{-(\ln x-\mu)^{2} / 2 \sigma^{2}} .
$$

The mean $\bar{x}$, mode $\hat{x}$, median $\tilde{x}$ and variance $\bar{\sigma}^{2}$ of $X$ are related to $\mu$ and $\sigma$ by way of the relations

$$
\begin{array}{ll}
\bar{x}=E[X]=e^{\mu+\sigma^{2} / 2} & , \quad \hat{x}=e^{\mu-\sigma^{2}} \\
\tilde{x}=e^{\mu} & , \quad \bar{\sigma}^{2}=\operatorname{var}[X]=\left(e^{\sigma^{2}}-1\right) e^{2 \mu+\sigma^{2}} .
\end{array}
$$

As illustrated in Figure 17, the mean, mode and median differ due to the skewed nature of the density. We note that for the normal density, $\mu$ and $\sigma$ have the same units as $x$ whereas the physical meaning of these units is lost in the lognormal relations (50).

The dependence of $f$ on $\mu$ and $\sigma$ is illustrated in Figure 18. Note that $X$ approaches a normal density with $\bar{x}=\tilde{x}=\hat{x}=e^{\mu}$ as $\sigma$ decreases and the density limits to the Dirac density at $e^{\mu}$ as $\sigma \rightarrow 0$.

As noted on page 9 of [1], the $q^{\text {th }}$ quantile of the lognormal density can be expressed as

$$
\left[e^{\mu-q \sigma}, e^{\mu-q \sigma}\right]
$$

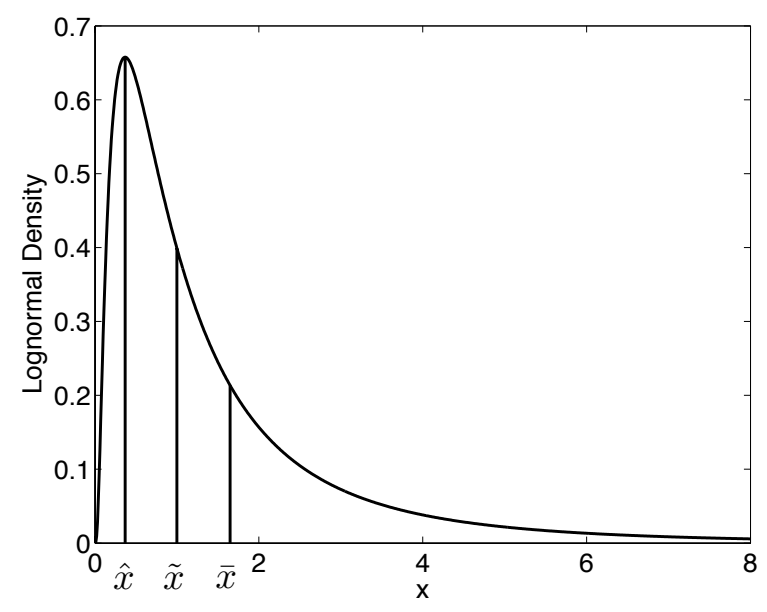

Figure 17: Mode $\hat{x}$, median $\tilde{x}$ and mean $\bar{x}$ of the lognormal density with $\mu=0, \sigma=1$. 
as illustrated in Figure 19. This relation, along with (50), will be used in Section 4.1 to specify initial density parameter values in terms of measured properties of polarization and magnetization data. For example, two standard deviations, which represents a probability of $94.5 \%$ is plotted for normal and lognormal densities in Figure 19.

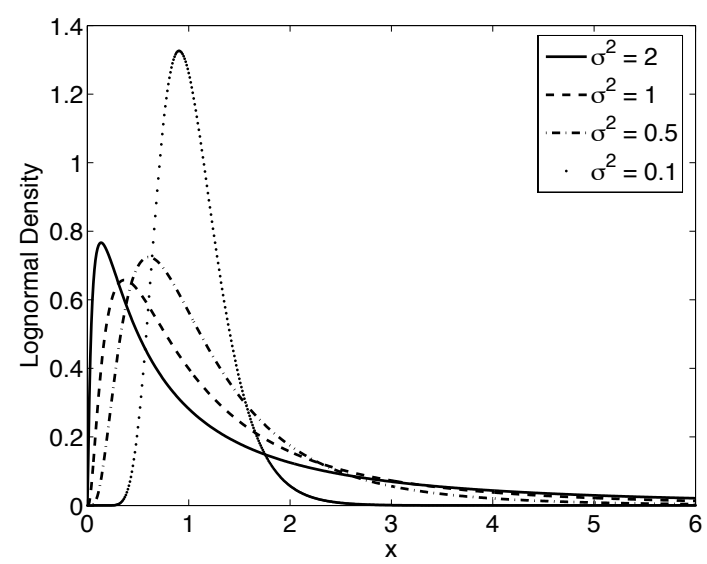

(a)

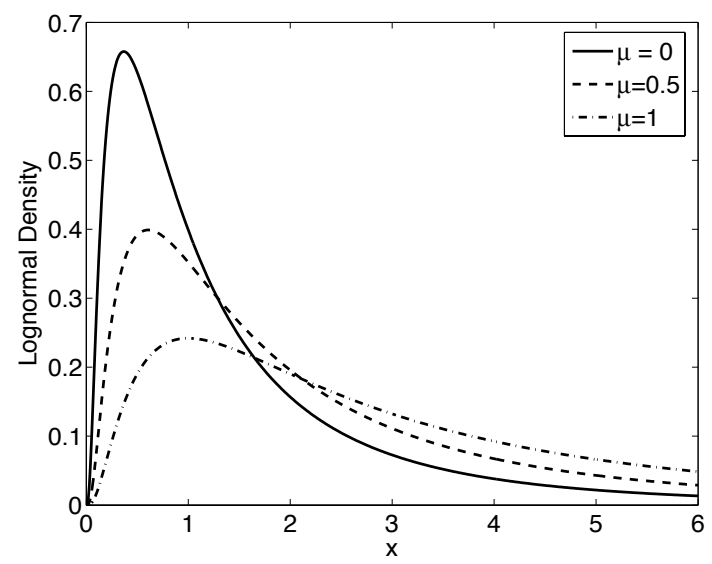

(b)

Figure 18: Dependence of the lognormal density on (a) $\sigma^{2}$ with $\mu=0$, and (b) $\mu$ with $\sigma^{2}=1$.

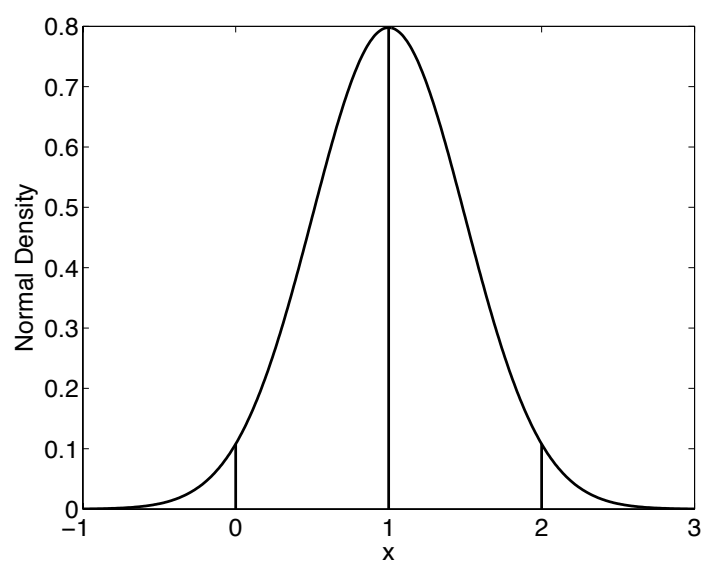

(a)

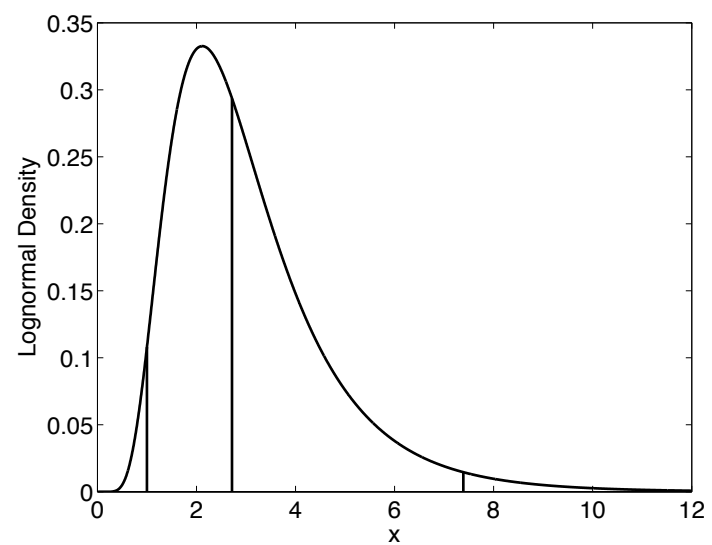

(b)

Figure 19: Median values and 94.5\% confidence intervals with $\mu=1, \sigma=0.5$ for a (a) normal density $[\mu-2 \sigma, \mu+2 \sigma]$, and (b) lognormal density $\left[e^{\mu-2 \sigma}, e^{\mu+2 \sigma}\right]$.

\section{Acknowledgements}

This research was supported in part by the Air Force Office of Scientific Research through the grants AFOSR FA9550-08-1-0348, AFOSR FA9550-11-10152 and the NSF grant NSF DMS-0636590 EMSW21-RTG. The authors gratefully thank Alex York for providing the PZT data used for validation in Section 5. We also thank John Crews for providing detailed feedback regarding the paper. 


\section{References}

[1] J. Aitchison and J.A.C. Brown, The Lognormal Distribution, Cambridge University Press, Cambridge, UK, 1963.

[2] K. Bhattacharya and G. Ravichandran, "Ferroelectric perovskites for electromechanical actuation," Acta Materialia, 51, pp. 5941-5960, 2003.

[3] T.R. Braun and R.C. Smith, "FPGA-based model implementation for real-time control of smart material systems operating in hysteretic regimes," Cansmart 2007, International Workshop on Smart Materials and Structures, Montreal, Quebec, CN, October 10-11, 2007.

[4] T.R. Braun and R.C. Smith, "High speed model implementation and inversion techniques for ferroelectric and ferromagnetic transducers," Journal of Intelligent Material Systems and Structures, 19(11), pp. 1295-1310, 2008.

[5] E. Burcsu, G. Ravichandran and K. Bhattacharya, "Large electrostrictive actuation of barium titanate single crystals," Journal of the Mechanics and Physics of Solids, 52, pp. 823-846, 2004.

[6] J.M. Ernstberger, High Speed Parameter Estimation for a Homogenized Energy Model, PhD Dissertation, North Carolina State University, Raleigh, NC, 2008.

[7] Z. Hu, R.C. Smith and J.M. Ernstberger, "Data driven techniques to estimate parameters in a rate-dependent ferromagnetic hysteresis model," Physica B, to appear.

[8] Z. Hu, R.C. Smith, M. Hays and W.S. Oates, "Statistical parameter estimation and uncertainty quantification for macro-fiber composites operating in nonlinear and hysteretic regimes," proceedings of the 50th IEEE Conference on Decision and Control and European Control Conference, Orlando, FL, December 12-15, pp. 2764-2769. 2011.

[9] J.E. Huber, "Micromechanical modelling of ferroelectrics," Current Opinion in Solid State and Materials Science, 9, pp. 100-106, 2005.

[10] B. Jaffe, W.R. Cook, Jr., and H. Jaffe, Piezoelectric Ceramics, Academic Press, New York, 1971.

[11] N.L. Johnson and S. Kotz, Continuous Univariate Distributions - I Distributions in Statistics, John Wiley and Sons, New York, 1970.

[12] S.-J. Kim and S. Seelecke, "A rate-dependent three-dimensional free energy model for ferroelectric single crystals," International Journal of Solids and Structures, 44, pp. 1196-1209, 2007.

[13] C.M. Landis, "Non-linear constitutive modeling of ferroelectrics," Current Opinion in Solid State and Materials Science, 8, pp. 59-69, 2004.

[14] C.S. Lynch, "The effect of uniaxial stress on the electro-mechanical response of 8/65/35 PLZT, Acta Materialia, 44(10), pp. 4137-4148, 1996.

[15] J. McMahon, J. Crews and R.C. Smith, "Approximate inversion techniques for the ferroelectric homogenized energy model for ferroelectric materials," preprint.

[16] C. Miehe and D. Rosato, "A rate-dependent incremental variational formulation of ferroelectricity," International Journal of Engineering Science, 49, pp. 466-496, 2011 
[17] C. Sherman and J.L. Butler, Transducers and Arrays for Underwater Sound, Springer Science and Business Media, New York, 2007.

[18] R.C. Smith, Smart Material Systems: Model Development, SIAM, Philadelphia, PA, 2005.

[19] R.C. Smith and Z. Hu, "The homogenized energy model (HEM) for characterizing polarization and strains in hysteretic ferroelectric materials: Material properties and uniaxial model development," Journal of Intelligent Material Systems and Structures, submitted.

[20] R.C. Smith, A.G. Hatch, B. Mukherjee and S. Liu, "A homogenized energy model for hysteresis in ferroelectric materials: General density formulation," Journal of Intelligent Material Systems and Structures, 16(9), pp. 713-732, 2005.

[21] R.C. Smith, S. Seelecke, Z. Ounaies and J. Smith, "A free energy model for hysteresis in ferroelectric materials," Journal of Intelligent Material Systems and Structures, 14(11), pp. 719-739, 2003.

[22] J.C. Spall, Introduction to Stochastic Search and Optimization, John Wiley and Sons, Hoboken, NJ, 2003.

[23] N. Uchida and T. Iketa, "Temperature and bias characteristics of $\mathrm{Pb}(\mathrm{Zr}, \mathrm{Ti}) \mathrm{O}_{3}$ families ceramics," Japanese Journal of Applied Physics, 4(11), pp. 867-880, 1965.

[24] A. York, Experimental characterization and modeling of electro-mechanically coupled ferroelectric actuators, PhD Dissertation, North Carolina State University, Raleigh, NC, 2008.

[25] A. York and S. Seelecke, "Experimental study of the electro-mechanical switching behavior of a piezoelectric stack actuator," Proceedings of the SPIE, 6929:692907, 2008.

[26] D. Zhou, M. Kamlah and D. Munz, "Rate dependence of soft PZT ceramics under electric field loading," Proceedings of the SPIE, 4333, pp. 64-70, 2001. 